\title{
OPTIMIZED SCHWARZ WAVEFORM RELAXATION FOR THE PRIMITIVE EQUATIONS OF THE OCEAN
}

\author{
E. AUDUSSE* ${ }^{*}$ P. DREYFUSS ${ }^{\dagger}$, AND B. MERLET. $\ddagger$
}

\begin{abstract}
In this article we are interested in the derivation of efficient domain decomposition methods for the viscous primitive equations of the ocean. We consider the rotating $3 \mathrm{~d}$ incompressible hydrostatic Navier-Stokes equations with free surface. Performing an asymptotic analysis of the system in the regime of small Rossby numbers, we compute an approximate Dirichlet to Neumann operator and build an optimized Schwarz waveform relaxation algorithm. We established that the algorithm is well defined and provide numerical evidences of the convergence of the method.
\end{abstract}

Key words. Domain Decomposition, Schwarz Waveform Relaxation Algorithm, Fluid Mechanics, Primitive Equations, Finite Volume Methods

AMS subject classifications. 65M55, 76D05, 76M12.

1. Introduction. A precise knowledge of ocean parameters (velocity, temperature...) is an essential tool to obtain climate and meteorological forecast. This task is nowadays of major importance and the need of global or regional simulations of the evolution of the ocean is strong. Moreover the large size of global simulations and the interaction between global and regional models require the introduction of efficient domain decomposition methods.

The evolution of the ocean is commonly modelized by the use of the viscous primitive equations. In our context, the primitive equations may be regarded as a refinement of the viscous shallow water equations. This last system describes the evolution of the barotropic velocity (the vertical average $\bar{U}$ of the velocity $U$ ) and of the water height. We consider here a 3D model that also predicts the evolution of the baroclinic velocities $U-\bar{U}$. This system is deduced from the full three dimensional incompressible Navier-Stokes equations with free surface simplified by the Boussinesq hypothesis and the hydrostatic approximation. It is implemented in all the major software that are concerned with global or/and regional simulations of ocean or/and atmosphere (we refer for example to NEMO, MOM or HYCOM for global models and ROMS or MARS for regional models).

The primitive equations have been studied for twenty years and important theoretical results are now available $[27,4]$. The numerical treatment of this system has been also strongly investigated [26]. But the key point here is to simulate global circulation on the earth for long time and/or with small space discretization. This type of computations can not be performed on a single computer in realistic CPU time and need to be parallelized. The problem is then to provide an efficient domain decomposition method.

We propose in this article to investigate these questions in the context of a quite recent efficient domain decomposition method : the Schwarz waveform relaxation type algorithms.

*LAGA, Université Paris Nord - Institut Galilée. Avenue J.B. Clément, 93430 Villetaneuse (audusse@math.univ-paris13.fr).

${ }^{\dagger}$ Laboratoire J. A. Dieudonné, Université de Nice. Parc Valrose - 06000, France (dreyfuss@unice.fr).

${ }^{\ddagger}$ LAGA, Université Paris Nord - Institut Galilée. Avenue J.B. Clément, 93430 Villetaneuse (merlet@math.univ-paris13.fr). 
In the oceanographic community, the related (but different) issue of finding Open Boundary Conditions received a wide attention. By using a regional model for the evolution of the ocean on a limited area, we would introduce artificial boundaries. In a ideal situation, outgoing waves should cross these boundaries and leave the computational domain as if no artificial boundary were present. Symmetrically, relevant data should be included from a global model into the regional model through the artificial boundaries. The aim of Open Boundary Conditions (see e.g: $[18,20,2]$ ) is to approach this ideal behavior. Notice that the informations are only transmitted from the global model to the regional model without feedback, so an efficient OBC need to be accurate. In the present paper, the situation is different since we consider an iterative method for which an equilibrium is reached through reciprocal exchanges between subdomains. An other difference is the sensitivity with respect to the discretization method. As pointed out in [16] different discretizations of the same OBC may lead to very different behaviors. Here we expect that our boundary conditions will lead to similar behaviors for both continuous and discretized models.

Domain decomposition techniques have been greatly developed over the last decades and our purpose is not to make an exhaustive presentation of these methods. We refer the reader to $[24,28]$ for a general presentation and we restrict ourselves to the description of Schwarz waveform relaxation method. It is a relatively new domain decomposition technique that has been successfully applied to different classes of equations. This type of algorithm is the result of the interaction between classical Schwarz domain decomposition techniques and waveform relaxation algorithms. The strength of the method is to be explicitly designed for evolution equations and to allow different strategies for the space time discretization in each subdomain. Moreover it is even possible to consider different models in each subdomain without modifying the architecture of the interaction.

The heart of the classical Schwarz method is to solve the problem on the whole domain thanks to an iterative procedure where subproblems are solved on each subdomain by using boundary conditions transmitted from the neighboring subdomains. This idea comes from the early work of Schwarz [25] where it were introduced to prove the well-posedness of a Poisson problem in some nontrivial domains. This method is designed for stationary problems and presents in its original version two main numerical drawbacks : it needs an overlap between subdomains and it converges slowly [19]. We refer to [9] for a complete presentation.

The extension to time evolution problems was performed separately at the end of the nineties by Gander [8,6] and Giladi \& Keller [12] and was named Schwarz waveform relaxation. The authors mixed the classical Schwarz approach with waveform relaxation techniques developed in the context of large system of ordinary differential equations [17]. The transmitted quantities between subproblems were of Dirichlet type. Optimized Schwarz waveform relaxation methods were developed with the introduction of more sophisticated (and more efficient) transmission conditions. These new choice of transmission conditions were based on previous works concerning absorbing boundary conditions $([7,13,14]$ respectively for hyperbolic, elliptic and incompletely parabolic equations). The same ideas were used to derive efficient transmission conditions between the subdomains : since the exact transparent conditions can not be implemented in general (it may lead to non-local pseudo-differential operators that are too costly to evaluate), the derivation of some approximate conditions is performed. These conditions can be optimized with respect to some free parameters 
which justifies the name of the method. The optimized Schwarz waveform relaxation method was first applied to the wave equation [11] and then to the advection-diffusion equation with constant or variable coefficients [21, 10]. More recently the method has been extended to the linearized viscous shallow water equations without advection term $[22,23]$. Here we are interested in the application of the method to the system of Primitive Equations of the ocean. It leads to non-trivial new problems (efficient transmission conditions, well-posedness and convergence of the algorithm...) that we address in this article.

In the above mentioned works, the optimal transmission conditions were obtained through the computation of the Dirichlet to Neumann operators associated to the subproblems. In the setting of the linearized Primitive Equations of the ocean, it is no longer possible to compute exactly the symbols of these operators. Therefore, it is necessary to perform approximations at the stage of the derivation of transmission conditions. Moreover, in the absence of exact formulas, it is hardly possible to assess the difference between the optimal and approximate transmission conditions through their symbols. This is the main novelty of this paper to show that even in this case it is possible to find efficient transmission conditions.

The outline of the paper is the following : in Section 2 we write the equations and we introduce the asymptotic regime that we consider. In Section 3 we derive an approximated Dirichlet to Neumann operator, and define the associated Schwarz waveform relaxation algorithm. In Section 4 we define a weak formulation of the problem on the whole domain and prove that it is well-posed in the natural functional spaces. In Section 5 we introduce a weak formulation for the Schwarz waveform relaxation algorithm and prove that each sub-problem solved in the algorithm is well-posed. Finally we present some numerical results in Sections 6 .

2. The set of equations. We first write the primitive equations of the ocean. Then we present the simplified system from which we are able to derive efficient transmission conditions.

2.1. The primitive equations of the ocean. We consider the primitive equations of the ocean on the domain $(x, y, z, t) \in \mathbf{R} \times \mathbf{R} \times[-H(x, y), \zeta(x, y, t)] \times \mathbf{R}^{+}$where $-H(x, y)$ denotes the topography of the ocean and $\zeta(x, y, t)$ denotes the altitude of the free surface of the ocean. The primitive equations are commonly written [5]

$$
\begin{aligned}
\partial_{t} U_{h}+U_{h} \cdot \nabla_{h} U_{h}-\nu \Delta U_{h}+\frac{2}{\rho_{0}} \vec{\Omega} \wedge U_{h}+\frac{1}{\rho_{0}} \nabla_{h} p & =0, \\
\nabla_{h} \cdot U_{h}+\partial_{z} w & =0, \\
\partial_{z} p & =-\rho g,
\end{aligned}
$$

where the unknowns are the $3 \mathrm{~d}$-velocity $\left(U_{h}, w\right)=(u, v, w)$ and the pressure $p$. The parameters are the density $\rho$, the gravity $g$ and the eddy viscosity $\nu$. These equations are supplemented by initial and boundary conditions. At initial time, we impose

$$
U_{h}(\cdot, 0)=U_{h, i} \quad \text { in } \Omega, \quad \zeta(\cdot, 0)=\zeta_{i} \quad \text { in } \omega,
$$

where the subscript letters $i$ means "initial". At the bottom of the ocean we impose a non-penetration condition and a friction law of Robin type $\left(\alpha_{b}>0\right)$

$$
U_{h}(-H) \cdot \nabla_{h}(H)-w(-H)=0, \quad \partial_{n} U_{t}(-H)+\alpha_{b} U_{t}(-H)=0,
$$


where $U_{t}$ stands for the tangential velocity and $n$ denotes the outward normal vector to the bottom of the ocean.

The free surface is transported by a kinematic boundary condition

$$
\partial_{t} \zeta+U_{h}(\zeta) \cdot \nabla_{h} \zeta-w(\zeta)=0 .
$$

The equilibrium of the stresses at the free surface implies

$$
\left[\sigma-\left(p-p_{a}\right) I d\right] \cdot \frac{1}{\sqrt{1+\left(\partial_{x} \zeta\right)^{2}+\left(\partial_{y} \zeta\right)^{2}}}\left(\begin{array}{c}
\partial_{x} \zeta \\
\partial_{y} \zeta \\
1
\end{array}\right)=0
$$

where $\sigma$ is the viscosity tensor and $p_{a}(x, y, t)$ denotes the atmospheric pressure.

2.2. A linearized hydrostatic model. In order to derive simple and efficient transmission conditions for the Schwarz waveform relaxation method we make some assumptions on this set of equations.

First we use the divergence-free condition (2.2) and the non-penetration condition (2.4) to write the vertical velocity $w$ as a function of the horizontal velocity $U_{h}$ and we use the hydrostatic assumption (2.3) to write the pressure $p$ as a function of the water height $\zeta$. The remaining unknowns in the system are the horizontal velocity $U_{h}$ and the water height $\zeta$. The set of equations (2.1)-(2.3) stands

$$
\begin{array}{r}
\partial_{t} U_{h}+U_{h} \cdot \nabla_{h} U_{h}-\nu \Delta U_{h}+f C U_{h}+g \nabla_{h} \zeta=0, \\
\partial_{t} \zeta+\nabla_{h} \cdot \int_{-H}^{\zeta} U_{h} d z=0,
\end{array}
$$

with

$$
C=\left(\begin{array}{cc}
0 & -1 \\
1 & 0
\end{array}\right), \quad \text { and } \quad f:=2 \vec{\Omega} \cdot e_{z}
$$

The first equation is written on the domain $\mathbf{R}_{x} \times \mathbf{R}_{y} \times[-H(x, y), \zeta(x, y, t)]_{z} \times \mathbf{R}_{t}^{+}$ while the second one is written on $\mathbf{R}_{x} \times \mathbf{R}_{y} \times \mathbf{R}_{t}^{+}$. We consider for simplicity a flat bottom and a constant atmospheric pressure. Then we linearize the problem around a constant state which corresponds to a horizontal velocity $U_{0}=\left(u_{0}, v_{0}\right)$ and a horizontal free surface located at $z=0$. It follows that the water height $\zeta$ is a small perturbation. In the sequel $U_{h}$ denotes the perturbation on the horizontal velocity. The linearized problem is given by

$$
\begin{array}{r}
\partial_{t} U_{h}+U_{0} \cdot \nabla_{h} U_{h}-\nu \Delta U_{h}+f C U_{h}+g \nabla_{h} \zeta=0, \\
\partial_{t} \zeta+H \nabla_{h} \cdot \bar{U}_{h}+U_{0} \cdot \nabla_{h} \zeta=0,
\end{array}
$$

where $\bar{U}_{h}=\left(\begin{array}{c}\bar{u} \\ \bar{v}\end{array}\right):=\frac{1}{H} \int_{-H}^{0} U_{h} d z$ denotes the mean horizontal velocity of the flow. This mean velocity is called barotropic velocity by the oceanographic community while the deviation $U_{h}-\bar{U}_{h}$ is called baroclinic velocity [5].

Note that the first equation (2.6) is now written in the fixed domain $\mathbf{R}_{x} \times \mathbf{R}_{y} \times$ $[-H, 0]_{z} \times \mathbf{R}_{t}^{+}$. The associated boundary conditions are

$$
\partial_{z} U_{h}(z=0)=0, \quad\left\{\partial_{z} U_{h}+\alpha_{b} U_{h}\right\}(z=-H)=0,
$$


where the boundary condition at $z=0$ is deduced from the equilibrium of the stresses at the free surface (2.5). Indeed, with the help of the linearization procedure, we first deduce that at first order $\partial_{z} U_{h}(z=\zeta) \simeq 0$ and then $\partial_{z} U_{h}(z=0) \simeq 0$ since we assume that $\zeta$ is small.

In order to derive the transmission conditions we assume $\alpha_{b}=0$ in the sequel. However in the definition of the Schwarz waveform relaxation algorithm and in the numerical simulations, the condition $\alpha_{b}>0$ will be supported.

2.3. Dimensionless system. We choose characteristic horizontal and vertical lengths (denoted $L$ and $H$ respectively) and a characteristic velocity $U$ for the problem. We then introduce the dimensionless quantities

$$
(x, y)=L(\tilde{x}, \tilde{y}), \quad t=(L / U) \tilde{t}, \quad \zeta=H \tilde{\zeta}, \quad z=H \tilde{z}, \quad U_{h}=U \widetilde{U_{h}}, \quad U_{0}=U \widetilde{U_{0}} .
$$

The spatial domains of computation are $\Omega=\mathbf{R}_{x} \times \mathbf{R}_{y} \times(-1,0)_{z}$ for the momentum equation and $\omega=\mathbf{R}_{x} \times \mathbf{R}_{y}$ for the continuity equation. We study both equations in the time interval $[0, T]$, where $T>0$ is fixed. Dropping the " " for a better readability, the system in dimensionless variables stands

$$
\begin{aligned}
\partial_{t} U_{h}+U_{0} \cdot \nabla_{h} U_{h}-\frac{1}{R e} \Delta_{h} U_{h}-\frac{1}{R e^{\prime}} \partial_{z}^{2} U_{h}+\frac{1}{\varepsilon} C U_{h}+\frac{1}{F r^{2}} \nabla_{h} \zeta & =0, \\
\partial_{z} U_{h}(x, y, 0, t)=\partial_{z} U_{h}(x, y,-1, t) & =0, \\
U_{h}(\cdot, 0) & =U_{h, i}, \\
\partial_{t} \zeta+U_{0} \cdot \nabla_{h} \zeta+\nabla_{h} \cdot \bar{U}_{h} & =0, \\
\zeta(\cdot, 0) & =\zeta_{i} .
\end{aligned}
$$

We have introduced the characteristic quantities : $\varepsilon:=U /(f L)$ (the Rossby number), Re $:=U L / \nu$ (the horizontal Reynolds number), $R e^{\prime}:=H^{2} / L^{2} R e$ (the vertical Reynolds number) and $F r:=U / \sqrt{g H}$ (the Froude number).

We choose to exhibit the Rossby number as a small parameter. This is justified by the fact that we are interested in long-time oceanographic circulations for which the Rossby number is typically of magnitude $10^{-2}$.

3. The optimized Schwarz waveform relaxation algorithm. We are now interested in finding efficient transmission conditions for equations (2.8)-(2.12). We first present the Schwarz waveform relaxation method. Then we derive the relations satisfied by the optimal transmission conditions. Since we are not able to solve these equations analytically, we perform an asymptotic analysis with respect to the Rossby number $\varepsilon$ in order to derive some approximated transmission conditions. Finally we present a related optimized Schwarz waveform relaxation algorithm.

3.1. The Schwarz waveform relaxation method. The heart of the method is the following. We first divide the computational domain into an arbitrary number of subdomains. Then we solve each sub-problem independently for the whole time interval. The interactions between neighboring subdomains are entirely contained in the boundary conditions. An iterative procedure is considered until a prescribed precision is reached. The advantages of the method are clear : the parallelization is almost optimal: at each step the sub-problems are solved independently, so the space-time discretization strategies (or even the models...) can be chosen independently on each subdomain. Moreover at the end of each step only a small amount of informations are exchanged: for each subdomain, this quantity is proportional to the number of 
(space and time) discretization nodes on its interfaces. The main drawback is related to the needed number of iterations : the method is efficient if it converges quickly (in two or three iterations typically). This requirement needs the derivation of efficient transmission conditions.

In the sequel we consider for simplicity two subdomains but the method extends to an arbitrary number of subdomains.

We begin with some notations. First we introduce the left and right spatial subdomains $\Omega^{-}$and $\Omega^{+}$defined by:

$$
\Omega^{-}:=(-\infty, 0)_{x} \times \mathbf{R}_{y} \times(-1,0)_{z}, \quad \Omega^{+}:=(0,+\infty)_{x} \times \mathbf{R}_{y} \times(-1,0)_{z},
$$

and their interface

$$
\Gamma=\{0\}_{x} \times \mathbf{R}_{y} \times(-1,0)_{z} \simeq \mathbf{R}_{y} \times(-1,0)_{z} .
$$

We also introduce the domains $\omega^{ \pm}:= \pm(0,+\infty)_{x} \times \mathbf{R}_{y}$ for the unknowns that do not depend on the $z$ variable, and their interface $\gamma:=\{0\}_{x} \times \mathbf{R}_{y} \simeq \mathbf{R}_{y}$.

Let $D$ be some spatial open domain and $T>0$ be a given real number. Then we will write $D_{T}$ to denote the cylindrical domain $D_{T}:=D \times(0, T)$.

We denote by $\mathrm{PE}$ the set of equations (2.8), (2.9) and (2.11) and $X:=\left(U_{h}, \zeta\right)$ stands for the solution of this system with associated initial conditions $X_{i}:=\left(U_{h, i}, \zeta_{i}\right)$.

Then the $n^{t h}$ iteration of the Schwarz waveform relaxation algorithm is defined as follows:

$$
\left\{\begin{array} { r l r l } 
{ \mathrm { PE } ( X _ { - } ^ { n + 1 } ) } & { = 0 } & { } & { \text { on } \Omega _ { T } ^ { - } , } \\
{ X _ { - } ^ { n + 1 } ( \cdot , 0 ) } & { = X _ { i } } & { } & { \text { on } \Omega ^ { - } , } \\
{ \mathcal { B } _ { - } X _ { - } ^ { n + 1 } } & { = \mathcal { B } _ { - } X _ { + } ^ { n } } & { } & { \text { on } \Gamma _ { T } , }
\end{array} \quad \left\{\begin{array}{rlrl}
\operatorname{PE}\left(X_{+}^{n+1}\right) & =0 & & \text { on } \Omega_{T}^{+} \\
X_{+}^{n+1}(\cdot, 0) & =X_{i} & & \text { on } \Omega^{+} \\
\mathcal{B}_{+} X_{+}^{n+1} & =\mathcal{B}_{+} X_{-}^{n} & & \text { on } \Gamma_{T}
\end{array}\right.\right.
$$

where the operators $\mathcal{B}_{ \pm}$contain the transmission conditions.

In the classical Schwarz waveform relaxation algorithm [8, 6], the transmitted quantities are of Dirichlet type and the operators $\mathcal{B}_{ \pm}$are thus chosen to be the identity operator. Note that in this case an overlap is needed in the definition of the subdomains.

In the sequel we are interested in deriving more efficient transmission conditions. The transmission conditions are said to be optimal if the algorithm converges in two iterations to the solution of the initial problem. These optimal transmission conditions involve the Dirichlet to Neumann operator associated with the PE on the subdomains $\Omega_{T}^{ \pm}$. We will see that we are not able to obtain an explicit formulation for these optimal conditions ; anyway these optimal boundary conditions are not local and consequently too expensive to be useful from a numerical point of view.

Recent methods have been developed in order to approximate these optimal conditions by analytical or numerical means - see the review paper [9] for elliptic problems and [10] for parabolic evolution equations.

Here we will perform an asymptotic analysis of the system with respect to the Rossby number $\varepsilon$ in order to deduce a set of efficient transmission conditions. This strategy has been initiated in $[22,23]$ for the shallow water equations without advection term. In our context, the barotropic velocities and the water height solve the linearized shallow water equations with advection term (see in Fourier-Laplace variables (3.6)(3.7)). The main difference with the situation considered in $[22,23]$ is the presence of this advection term. Let us list some consequences. First, due to the advection term, we need 
to supplement the continuity equation with boundary conditions on interfaces with incoming characteristics. These new boundary conditions will be optimized together with the transmission conditions for the momentum equations. Second, (3.6)(3.7) is a system of two second order ODEs in $x$ (momentum equations) and one first order ODE (continutiy equation). If we want to compute the Fourier-Laplace symbols of the corresponding Dirichlet to Neumann operators we need to explicit the solutions of a a family of ordinary differential equations of degree 5 which is not possible in general (and in our case). On the contrary, if the advection velocity is set to zero, the continuity equation does not contain any $x$ derivative so in [23], the author is able to compute explicitly the symbols of the Dirichlet to Neumann operators. Third, in [23], the author perform an asymptotic analysis in the limit of vanishing Coriolis forces (equatorial approximation) corresponding to large Rossby numbers whereas, in the present paper, the flow is driven by Coriolis forces (small Rossby number $\varepsilon$ ). The two asymptotics lead to very different transmission conditions (see Remark 3.4 below)."

Let us first describe in a formal setting the ideal case of optimal transmission conditions for the Schwarz waveform relaxation algorithm (3.1). We consider the case $u_{0}>0$. The case $u_{0}<0$ is deduced by applying the symmetry " $x^{\prime}=-x$ ". Integrating the linearized Primitive equations PE on a subdomain, we see that the flux of the unknown $\left(U_{h}, \zeta\right)$ through the interface $\Gamma$ is given by

$$
\left(\frac{1}{R e} \partial_{x} U_{h}-u_{0} U_{h}-\frac{1}{F r^{2}}\left(\begin{array}{l}
\zeta \\
0
\end{array}\right), u_{0} \zeta+\bar{u}\right)
$$

Using this flux as a Neumann operator, we define the Dirichlet to Neumann operators as follows. Consider a Dirichlet data $X_{b}=\left(U_{h, b}, \zeta_{b}\right)$, we set

$$
\mathcal{S}_{-}^{U_{h}} X_{b}:=\left(\frac{1}{R e} \partial_{x} U_{h}-u_{0} U_{h}-\frac{1}{F r^{2}}\left(\begin{array}{l}
\zeta \\
0
\end{array}\right)\right)_{\mid \Gamma_{T}},
$$

where $X=\left(U_{h}, \zeta\right)$ solves the homogeneous system $\mathrm{PE}(X)=0$ on $\Omega_{T}^{+}$with vanishing iniatial condition $X(\cdot, 0)=0$ on $\Omega^{+}$and boundary condition $X=X_{b}$ on $\Gamma_{T}$. Symmetrically, consider a Dirichlet data $U_{h, b}$, we set

$$
\left(\mathcal{S}_{+}^{U_{h}} X_{b}, \mathcal{S}_{+}^{\zeta} X_{b}\right):=\left(\frac{1}{R e} \partial_{x} U_{h}-u_{0} U_{h}-\frac{1}{F r^{2}}\left(\begin{array}{l}
\zeta \\
0
\end{array}\right), u_{0} \zeta+\bar{u}\right)_{\mid \Gamma_{T}},
$$

where $X=\left(U_{h}, \zeta\right)$ solves $\operatorname{PE}(X)=0$ on $\Omega_{T}^{-}$with initial condition $X(\cdot, 0)=0$ on $\Omega^{-}$ and boundary condition $U_{h}=\bar{U}_{h}$ on $\Gamma_{T}$.

Notice that since we consider the case $u_{0}>0$, the third boundary condition is only relevant in the subdomain $\Omega_{T}^{+}$. This is why in the later case we do not have to prescribe a boundary condition for $\zeta$.

Once these Dirichlet to Neumann operators are defined we can introduce the optimal transmission conditions

$$
\begin{aligned}
& \mathcal{B}_{-} X=\left(\frac{1}{R e} \partial_{x} U_{h}-u_{0} U_{h}-\frac{1}{F r^{2}}\left(\begin{array}{l}
\zeta \\
0
\end{array}\right)-\mathcal{S}_{-}^{U_{h}} X\right) \\
& \mathcal{B}_{+} X={ }^{t}\left(-\frac{1}{R e} \partial_{x} U_{h}+u_{0} U_{h}+\frac{1}{F r^{2}}\left(\begin{array}{l}
\zeta \\
0
\end{array}\right)-\mathcal{S}_{+}^{U_{h}} X, u_{0} \zeta+\bar{u}-\mathcal{S}_{+}^{\zeta} X\right),
\end{aligned}
$$


Proposition 3.1. With this particular choice of transmission operators $\mathcal{B}_{ \pm}$, the algorithm (3.1) converges in two iterations.

Proof. By linearity, we may assume that the exact solution is $0\left(X_{i} \equiv 0\right)$. At the initial step the solutions on each subdomain do not satisfy any particular property. But the first iterate solves the primitive equations with vanishing initial conditions. It follows from the very definition of the operators $\mathcal{S}_{ \pm}$that in the definition of the second iterate, the right hand sides of the transmission conditions vanish for both sub-problems. Consequently, the second iterates vanish.?

The operators (3.2)(3.3) being non-local pseudo-differential operators, they are not well suited for numerical implementation. Our strategy is to approximate these operators by numerically cheap operators. Of course the two-step convergence property will be lost. The quality of the approximation will be measured through the convergence rate of the algorithm. From the structure of $(3.2)(3.3)$, we choose to write $\mathcal{B}_{ \pm}$ as perturbations of the natural operators transmitted through the interface:

$$
\begin{aligned}
& \mathcal{B}_{-} X=\left(\frac{1}{R e} \partial_{x} U_{h}-u_{0} U_{h}-\frac{1}{F r^{2}}\left(\begin{array}{c}
\zeta \\
0
\end{array}\right)-\tilde{\mathcal{S}}_{-}^{U_{h}} X\right) \\
& \mathcal{B}_{+} X=\left(-\frac{1}{R e} \partial_{x} U_{h}+u_{0} U_{h}+\frac{1}{F r^{2}}\left(\begin{array}{c}
\zeta \\
0
\end{array}\right)-\tilde{\mathcal{S}}_{+}^{U_{h}} X, u_{0} \zeta+\bar{u}-\tilde{\mathcal{S}}_{+}^{\zeta} X\right)^{t},
\end{aligned}
$$

where $\tilde{\mathcal{S}}_{ \pm}^{U_{h}}$ and $\tilde{\mathcal{S}}_{+}^{\zeta}$ are pseudo-differential operators that will in some sense approximate the Dirichlet to Neumann operators.

Let us finally remark that the differences in the expression of the two transmission operators $\mathcal{B}_{ \pm}$are due to the sign $u_{0}>0$. Since $\mathcal{B}_{-}$contains the information that is transmitted from $\Omega_{T}^{+}$to $\Omega_{T}^{-}$it is constructed on three boundary values (velocities and water height) but it has to transmit only two boundary conditions for momentum equations (2.8). On the contrary $\mathcal{B}_{+}$is constructed on two boundary values (velocities) but has to send three boundary conditions (for momentum and continuity equations).

In the next subsections we will identify optimal and approximated transmission operators. To carry out the computation of the Dirichlet to Neumann operators we perform Fourier-Laplace transforms.

3.2. Laplace-Fourier transform of the primitive equations. We perform a Fourier transform in the $y$ variable and a Laplace transform in time on the equations (2.8)-(2.11). The dual variables are respectively denoted $\eta \in \mathbf{R}$ and $s=\sigma+i \tau \in$ C. The real part $\sigma$ is assumed to be strictly positive. We obtain in each subdomain the same set of differential equations

$$
\begin{array}{r}
\left\{s+u_{0} \partial_{x}+i \eta v_{0}-\frac{1}{R e} \partial_{x}^{2}+\frac{1}{R e} \eta^{2}-\frac{1}{R e^{\prime}} \partial_{z}^{2}+\frac{1}{\varepsilon} B\right\} \hat{U}_{h}+\frac{1}{F r^{2}}\left(\begin{array}{c}
\partial_{x} \\
i \eta
\end{array}\right) \hat{\zeta}=0 \\
\left\{s+u_{0} \partial_{x}+i \eta v_{0}\right\} \hat{\zeta}+\partial_{x} \hat{\bar{u}}+i \eta \hat{\bar{v}}=0 .
\end{array}
$$

In the $z$ direction we introduce the eigenmodes of the operator $-\partial_{z}^{2}$ on $(-1,0)$ with homogeneous Neumann boundary conditions (2.9)

$$
\boldsymbol{e}_{n}(z):=\alpha_{n} \cos \left(\mu_{n} z\right) \quad \text { with } \quad \mu_{n}:=n \pi ; \quad \alpha_{0}:=1 \quad \text { and } \quad \alpha_{n}:=\sqrt{2} \quad \text { if } \quad n>0 .
$$


Then we search for the solution of the form

$$
\hat{U}_{h}(x, z)=\sum_{n=0}^{\infty} \hat{U}_{h}^{n}(x) e_{n}(z) .
$$

Note that we obviously have $\hat{\bar{U}}_{h}=\hat{U}_{h}^{0}$. So the first vertical mode $\hat{U}_{h}^{0}$ represents the barotropic velocity while the sum of the other ones denotes the baroclinic deviation.

The barotropic mode is coupled with the water height and it is the solution of the following system of three ordinary differential equations,

$$
\begin{aligned}
-\frac{1}{R e} \partial_{x}^{2} \hat{U}_{h}^{0}+u_{0} \partial_{x} \hat{U}_{h}^{0}+\left\{s+i \eta v_{0}+\frac{1}{R e} \eta^{2}+\frac{1}{\varepsilon} B\right\} \hat{U}_{h}^{0}+\frac{1}{F r^{2}}\left(\begin{array}{c}
\partial_{x} \hat{\zeta} \\
i \eta \hat{\zeta}
\end{array}\right)=0 \\
u_{0} \partial_{x} \hat{\zeta}+\left(s+i \eta v_{0}\right) \hat{\zeta}+\partial_{x} \hat{u}^{0}+i \eta \hat{v}^{0}=0
\end{aligned}
$$

This last system is exactly the Laplace-Fourier transform of the so-called linearized viscous shallow water equations.

For the other vertical modes we have a set of reaction advection diffusion systems

$$
-\frac{1}{R e} \partial_{x}^{2} \hat{U}_{h}^{n}+u_{0} \partial_{x} \hat{U}_{h}^{n}+\left\{s+i \eta v_{0}+\frac{1}{R e} \eta^{2}+\frac{1}{R e^{\prime}} \mu_{n}^{2}+\frac{1}{\varepsilon} B\right\} \hat{U}_{h}^{n}=0 .
$$

REMARK 3.2. Here we expand the solution in a series of known modes solving independent equations. This is not a generic situation. In particular if we set $\alpha_{b}>0$ in (2.7) then the Laplace-Fourier transform does not lead to any decoupling of the equations. Indeed, in the momentum equations, the vertical operator $\partial_{z}^{2}$ is supplemented with the boundary conditions $\partial_{z} U_{h}(0)=0$ and $\left(\partial_{z}+\alpha_{b}\right) U_{h}(-1)=0$, so if $\alpha_{b}>0$, constant functions are not eigenvectors of the vertical operator. On the other hand the (source) term $\nabla_{h} \zeta$ is constant in the vertical direction $(\zeta=\zeta(x, y, t))$. Consequently, there is no nice decoupling of the momentum equations in vertical modes: all these modes are coupled through the continuity equation.

Notice that we only use the friction-free assumption for deriving the transmission conditions. For the well-posedness and the numerical study of the algorithm, we will consider the general situation $\alpha_{b} \geq 0$.

3.3. Optimal transmission conditions for the baroclinic modes. The derivation of optimal transmission conditions for an advection diffusion equation was performed in [21]. Here, since we deal with systems, the situation is more complex, . The baroclinic modes are not coupled with the evolution of the water height. Hence for these modes the transmission operators have only two components:

$$
\mathcal{B}_{ \pm}^{n}=\left(\mp \frac{1}{R e} \partial_{x} U_{h} \pm u_{0} U_{h}-\mathcal{S}_{ \pm}^{U_{h}, n}\right) .
$$

We search for the solution of system (3.8) as a sum of exponentials $x \mapsto e^{\lambda x}$. Substituting this ansatz in the system, we obtain that $e^{\lambda x}$ solves (3.8) if and only if $\lambda$ is a root of the determinant of the matrix

$$
M_{n}(\lambda):=\left(\begin{array}{cc}
-\frac{\lambda^{2}}{R e}+u_{0} \lambda+s+\frac{\eta^{2}}{R e}+\frac{\mu_{n}^{2}}{R e^{\prime}}+i \eta v_{0} & -\frac{1}{\varepsilon} \\
\frac{1}{\varepsilon} & -\frac{\lambda^{2}}{R e}+u_{0} \lambda+s+\frac{\eta^{2}}{R e}+\frac{\mu_{n}^{2}}{R e^{\prime}}+i \eta v_{0}
\end{array}\right) .
$$


This determinant is a polynomial of degree four in $\lambda$ and we can compute its roots

$$
\begin{aligned}
& \qquad \lambda_{ \pm}^{n,+}:=\frac{R e}{2}\left(u_{0}+\sqrt{\Delta_{ \pm}^{n}}\right), \quad \lambda_{ \pm}^{n,-}:=\frac{R e}{2}\left(u_{0}-\sqrt{\Delta_{ \pm}^{n}}\right) \\
& \text { where } \\
& \qquad \Delta_{ \pm}^{n}:=u_{0}^{2}+\frac{4}{R e}\left(\frac{\eta^{2}}{R e}+\frac{\mu_{n}^{2}}{R e^{\prime}}+s+i \eta v_{0} \pm \frac{i}{\varepsilon}\right) .
\end{aligned}
$$

Every solution $\lambda_{ \pm}^{n, \pm}$ is associated with a one dimensional kernel generated by the vector $\phi_{ \pm}^{n, \pm}$ defined by

$$
\phi_{+}^{n, \pm}=\left(\begin{array}{c}
1 \\
-i
\end{array}\right), \quad \phi_{-}^{n, \pm}=\left(\begin{array}{l}
1 \\
i
\end{array}\right)
$$

Since the solutions must vanish at infinity we search for solutions in $\Omega^{-}$of the form

$$
\hat{U}_{h,-}^{n}(x)=\alpha_{+}^{n,+} e^{\lambda_{+}^{n,+} x} \phi_{+}^{n,+}+\alpha_{-}^{n,+} e^{\lambda_{-}^{n,+} x} \phi_{-}^{n,+}=\Phi^{n,+} \cdot \exp \left(x \Lambda^{n,+}\right) \cdot \alpha^{n,+}
$$

where

$$
\Phi^{n, \pm}:=\left(\begin{array}{cc}
1 & 1 \\
-i & i
\end{array}\right), \quad \Lambda^{n, \pm}:=\left(\begin{array}{cc}
\lambda_{+}^{n, \pm} & 0 \\
0 & \lambda_{-}^{n, \pm}
\end{array}\right), \quad \alpha^{n, \pm}:=\left(\begin{array}{c}
\alpha_{+}^{n, \pm} \\
\alpha_{-}^{n, \pm}
\end{array}\right) .
$$

In $\Omega^{+}$we search for the solution of the form

$$
\hat{U}_{h,+}^{n}(x)=\alpha_{+}^{n,-} e^{\lambda_{+}^{n,-} x} \phi_{+}^{n,-}+\alpha_{-}^{n,-} e^{\lambda_{-}^{n,-} x} \phi_{-}^{n,-}=\Phi^{n,-} \cdot \exp \left(x \Lambda^{n,-}\right) \cdot \alpha^{n,-} .
$$

It follows from relations (3.11) and (3.13) that

$$
\partial_{x} \hat{U}_{h, \mp}^{n}(x)=\Phi^{n, \pm} \cdot \Lambda^{n, \pm} \cdot \exp \left(x \Lambda^{n, \pm}\right) \cdot \alpha^{n, \pm}=\Phi^{n, \pm} \cdot \Lambda^{n, \pm} \cdot\left[\Phi^{n, \pm}\right]^{-1} \cdot \hat{U}_{h, \mp}^{n} \cdot
$$

We can now define the operator $\mathcal{S}_{ \pm}^{u, n}$ in (3.9) in order to derive an optimal algorithm. This is done through its Laplace-Fourier symbol:

$$
\hat{\mathcal{S}}_{ \pm}^{u, n}:=\mp \frac{1}{R e} \Phi^{n, \pm} \Lambda^{n, \pm}\left[\Phi^{n, \pm}\right]^{-1} \pm u_{0} \text { Id }
$$

3.4. Approximate transmission conditions for baroclinic modes. Since we want to construct an efficient but simple Schwarz waveform relaxation algorithm we will derive approximate transmission conditions by considering an asymptotic analysis of the results of the previous subsection.

The definition (3.10) of $\Delta_{ \pm}^{n}$ leads to the expansion $\hat{\mathcal{S}}_{ \pm}^{U_{h}, n}=\hat{\tilde{\mathcal{S}}}_{ \pm}^{U_{h}, n}+O(\sqrt{\varepsilon})$ with

$$
\hat{\tilde{\mathcal{S}}}_{ \pm}^{U_{h}, n}:=\frac{1}{2}\left(\begin{array}{cc} 
\pm u_{0}-\sqrt{\frac{2}{R e}} \frac{1}{\sqrt{\varepsilon}} & \sqrt{\frac{2}{R e}} \frac{1}{\sqrt{\varepsilon}} \\
-\sqrt{\frac{2}{R e}} \frac{1}{\sqrt{\varepsilon}} & \pm u_{0}-\sqrt{\frac{2}{R e}} \frac{1}{\sqrt{\varepsilon}}
\end{array}\right) .
$$

Note that the approximated operator (3.14) does not depend on $n$. Consequently the related approximated transmission operators (3.9) can be applied to the whole baroclinic velocity, i.e. to the sum of the baroclinic modes. 
3.5. Approximate transmission conditions for the barotropic mode. The derivation of optimal transmission conditions for the linearized viscous shallow water equations without advection term was performed in [23]. Here we are interested in the linearized viscous shallow water equations (3.6)-(3.7). We will search for transmission operators of the form (3.4)-(3.5).

As for the baroclinic modes we search for the solution of system (3.6)-(3.7) as a sum of exponentials $e^{\lambda x}$. Here $\lambda$ has to be a root of the determinant of the matrix $M_{0}(\lambda)$ defined by

$$
\left(\begin{array}{ccc}
-\frac{\lambda^{2}}{R e}+u_{0} \lambda+s+\frac{\eta^{2}}{R e}+i \eta v_{0} & -\frac{1}{\varepsilon} & \lambda / F r^{2} \\
\frac{1}{\varepsilon} & -\frac{\lambda^{2}}{R e}+u_{0} \lambda+s+\frac{\eta^{2}}{R e}+i \eta v_{0} & \frac{i \eta}{F r^{2}} \\
\lambda & i \eta & s+u_{0} \lambda+i \eta v_{0}
\end{array}\right)
$$

This determinant is a polynomial of degree five which does not admit a trivial decomposition. Hence it is not possible to derive an explicit formula for the solutions of (3.6)-(3.7). Consequently, we are not able to obtain an explicit form for the optimal transmission conditions for the barotropic mode, even in Fourier-Laplace variables.

Remark 3.3. To go further in the determination of the Dirichlet to Neumann operators, we could compute numerical approximations of the roots of the family of polynomials $p_{s, \eta}(\lambda):=\operatorname{det}\left(M_{0}(\lambda)\right)$ for a large range of values of $s, \eta$ and then find numerical approximations of the corresponding kernels. This method was proposed in [29] in the context of discrete transparent boundary conditions. However, in our case, the Dirichlet to Neumann operators are not differential operators and some approximations will be necessary for building useful transmission conditions (through e.g. Taylor expansion as in the related work [23]).

In order to derive approximated transmission conditions we use the fact that the Rossby number is a small parameter to compute approximated values of the roots of the determinant of $M_{0}(\lambda)$. The related approximated transmission conditions will be consistent with the results of the previous subsection for the baroclinic modes.

Since $u_{0}$ is positive we first notice that three roots (3.15)-(3.16) have a negative real part and two roots (3.17) have a positive real part. The negative roots will be denoted $\lambda_{ \pm}^{0,-}$ and $\lambda_{0}^{0}$. The positive ones will be denoted $\lambda_{ \pm}^{0,+}$. The notations for the related quantities that we introduce later are consistent with the previous ones (3.12). As above, we search for the solution in $\Omega^{-}$of the form

$$
\hat{X}_{-}(x)=\alpha_{+}^{0,+} e^{\lambda_{+}^{0,+} x} \phi_{+}^{0,+}+\alpha_{-}^{0,+} e^{\lambda_{-}^{0,+} x} \phi_{-}^{0,+}=: \Phi^{0,+} \cdot \exp \left(x \Lambda^{0,+}\right) \cdot \alpha^{0,+} .
$$

In $\Omega^{+}$we search for the solution of the form

$$
\hat{X}_{+}(x)=\alpha_{+}^{0,-} e^{\lambda_{+}^{0,-} x} \phi_{+}^{0,-}+\alpha_{-}^{0,-} e^{\lambda_{-}^{0,-} x} \phi_{-}^{0,-}+\alpha_{0}^{0} e^{\lambda_{0}^{0} x} \phi_{0}^{0}=: \Phi^{0,-} \cdot \exp \left(x \Lambda^{0,-}\right) \cdot \alpha^{0,-} .
$$


We compute the following approximations for the roots of the determinant of $M_{0}(\lambda)$ :

$$
\begin{aligned}
\lambda_{0}^{0} & =-\frac{s+i \eta v_{0}}{u_{0}}+O\left(\varepsilon^{2}\right), \\
\lambda_{ \pm}^{0,-} & =-\frac{\sqrt{ \pm i R e}}{\sqrt{\varepsilon}}+\left(\frac{R e u_{0}}{2}-\frac{R e}{4 F r^{2} u_{0}}\right)+O(\sqrt{\varepsilon}), \\
\lambda_{ \pm}^{0,+} & =\frac{\sqrt{ \pm i R e}}{\sqrt{\varepsilon}}+\left(\frac{\operatorname{Re} u_{0}}{2}-\frac{R e}{4 F r^{2} u_{0}}\right)+O(\sqrt{\varepsilon}) .
\end{aligned}
$$

The associated kernel is always one dimensional and spanned by:

$$
\begin{aligned}
\Phi_{0}^{0}= & (0,0,1)^{t}+O\left(\varepsilon^{2}\right) \\
\Phi_{ \pm}^{0,-}= & \left(u_{0} \mp \frac{i \sqrt{2 R e}}{4 F r^{2}} \sqrt{\varepsilon}, \mp i u_{0} \pm \frac{i \sqrt{2 R e}}{4 F r^{2}} \sqrt{\varepsilon}\right. \\
& \left.-1+\left\{\frac{ \pm \sqrt{2}}{4} \frac{i \sqrt{R e}}{u_{0} F r^{2}}-\frac{\sqrt{2}}{2} \frac{1 \mp i}{\sqrt{R e}}\left(\left( \pm u_{0}+i v_{0}\right) \eta+s\right)\right\} \sqrt{\varepsilon}\right)^{t}+O(\varepsilon), \\
\Phi_{ \pm}^{0,+}= & \left(u_{0} \pm \frac{i \sqrt{2 R e}}{4 F r^{2}} \sqrt{\varepsilon}, \mp i u_{0} \mp \frac{i \sqrt{2 R e}}{4 F r^{2}} \sqrt{\varepsilon},\right. \\
& \left.-1-\left\{\frac{ \pm \sqrt{2}}{4} \frac{i \sqrt{R e}}{u_{0} F r^{2}}-\frac{\sqrt{2}}{2} \frac{1 \mp i}{\sqrt{R e}}\left(\left(\mp u_{0}+i v_{0}\right) \eta+s\right)\right\} \sqrt{\varepsilon}\right)^{t}+O(\varepsilon) .
\end{aligned}
$$

As in the baroclinic modes case, we compute the approximated transmission operators in Laplace-Fourier variables by

$$
\begin{aligned}
& \hat{\tilde{\mathcal{S}}}_{-}^{U_{h}, 0}=\frac{1}{R e}\left[\Phi^{0,-} \Lambda^{0,-}\left[\Phi^{0,-}\right]^{-1}\right]_{2,3}-\left(\begin{array}{ccc}
u_{0} & 0 & -\frac{1}{R e} \\
0 & u_{0} & 0
\end{array}\right), \\
& \hat{\tilde{\mathcal{S}}}_{+}^{U_{h}, 0}=-\frac{1}{R e} \Phi^{0,+} \Lambda^{0,+}\left[\Phi^{0,+}\right]^{-1}+u_{0} I d
\end{aligned}
$$

where $M_{2,3}$ denotes the $2 \times 3$ matrix $\left(M_{i, j}\right)_{1 \leq i \leq 2,1 \leq j \leq 3}$ extracted from the $3 \times 3$ matrix $M$. It leads to the following Laplace-Fourier symbols

$$
\begin{gathered}
\hat{\tilde{\mathcal{S}}}_{-}^{U_{h}, 0}=\frac{1}{2}\left(\begin{array}{ccc}
-\frac{\sqrt{2}}{\sqrt{R e \varepsilon}}-u_{0}-\frac{1}{F r^{2} u_{0}} & \frac{\sqrt{2}}{\sqrt{R e \varepsilon}}+\frac{1}{2 F r^{2} u_{0}} & \frac{-2}{F r^{2}} \\
-\frac{\sqrt{2}}{\sqrt{R e \varepsilon}}+\frac{1}{2 F r^{2} u_{0}} & -\frac{\sqrt{2}}{\sqrt{R e \varepsilon}}-u_{0} & 0
\end{array}\right), \\
\hat{\tilde{\mathcal{S}}}_{-}^{U_{h}, 0}=\frac{1}{2}\left(\begin{array}{ccc}
-\frac{\sqrt{2}}{\sqrt{R e \varepsilon}}+u_{0}-\frac{1}{F r^{2} u_{0}} & \frac{\sqrt{2}}{\sqrt{R e \varepsilon}}-\frac{1}{2 F r^{2} u_{0}} \\
-\frac{\sqrt{2}}{\sqrt{R e \varepsilon}}-\frac{1}{2 F r^{2} u_{0}} & -\frac{\sqrt{2}}{\sqrt{R e \varepsilon}}+u_{0} \\
0 & 0
\end{array}\right) .
\end{gathered}
$$

By using relations (3.14), (3.18) and (3.19), we notice that

$$
\left[\hat{\tilde{\mathcal{S}}}_{ \pm}^{U_{h}, 0}\right]_{2,2}=\hat{\tilde{\mathcal{S}}}_{ \pm}^{U_{h}, n}+\left(\begin{array}{cc}
-\frac{1}{2 F r^{2} u_{0}} & \mp \frac{1}{4 F r^{2} u_{0}} \\
\mp \frac{1}{4 F r^{2} u_{0}} & 0
\end{array}\right)
$$


It follows that a part of the transmission conditions will be applied to the whole velocity while a second part will be applied only to the barotropic mode.

3.6. The optimized Schwarz waveform relaxation algorithm. Thanks to the computed approximated operators (3.14), (3.18) and (3.19) we can now derive an approximated Schwarz waveform relaxation algorithm for the linearized primitive equations (2.8)-(2.12).

Since the computed operators (3.14), (3.18) and (3.19) do not depend on $(\eta, s)$, the related operators in the real space are identical to their Laplace-Fourier symbols. It follows that the approximate transmission operators $\mathcal{B}_{ \pm}(3.4)-(3.5)$ have the following form

$$
\begin{gathered}
\mathcal{B}_{-} X=\left(\begin{array}{c}
\frac{1}{R e} \partial_{x} u+\left(\frac{\sqrt{2}}{2 \sqrt{R e \varepsilon}}-\frac{u_{0}}{2}\right) u-\frac{\sqrt{2} v}{2 \sqrt{R e \varepsilon}}+\frac{\bar{u}-\bar{v} / 2}{2 F r^{2} u_{0}} \\
\left.\frac{1}{R e} \partial_{x} v+\left(\frac{\sqrt{2}}{2 \sqrt{R e \varepsilon}}-\frac{u_{0}}{2}\right) v+\frac{\sqrt{2} u}{2 \sqrt{R e \varepsilon}}-\frac{\bar{u}}{4 F r^{2} u_{0}}\right) \\
\mathcal{B}_{+} X=\left(\begin{array}{c}
-\frac{1}{R e} \partial_{x} u+\frac{\zeta}{F r^{2}}+\left(\frac{\sqrt{2}}{2 \sqrt{R e \varepsilon}}+\frac{u_{0}}{2}\right) u-\frac{\sqrt{2} v}{2 \sqrt{R e \varepsilon}}+\frac{\bar{u}+\bar{v} / 2}{2 F r^{2} u_{0}} \\
-\frac{1}{R e} \partial_{x} v+\left(\frac{\sqrt{2}}{2 \sqrt{R e \varepsilon}}+\frac{u_{0}}{2}\right) v+\frac{\sqrt{2} u}{2 \sqrt{R e \varepsilon}}+\frac{\bar{u}}{4 F r^{2} u_{0}} \\
u_{0} \zeta+\bar{u}
\end{array}\right),
\end{array}\right.
\end{gathered}
$$

for which we recall that $\bar{u}$ and $\bar{v}$ represent the mean-values with respect to the $z$ variable of the velocities $u$ and $v$.

REMARK 3.4. In the limit of small $\varepsilon$, the momentum equation is driven by the zero-order operator $\varepsilon^{-1} C$. So, at leading order, the symbol of the Dirichlet to Neumann operator is also a zero-order operator. By contrast, in [23], the Rossby number $\varepsilon$ is treated as a large parameter and the leading term of the expansion involve time derivatives.

Note that by replacing the first component $\left(\mathcal{B}_{+} X\right)_{1}$ by the linear combination

$$
\left(\mathcal{B}_{+} X\right)_{1}-1 /\left(F r^{2} u_{0}\right)\left(\mathcal{B}_{+} X\right)_{3},
$$

we replace (3.21) by the equivalent transmission operators

$$
\widetilde{\mathcal{B}}_{+} X=\left(\begin{array}{c}
-\frac{1}{R e} \partial_{x} u+\left(\frac{\sqrt{2}}{2 \sqrt{R e \varepsilon}}+\frac{u_{0}}{2}\right) u-\frac{\sqrt{2} v}{2 \sqrt{R e \varepsilon}}-\frac{\bar{u}-\bar{v} / 2}{2 F r^{2} u_{0}} \\
-\frac{1}{R e} \partial_{x} v+\left(\frac{\sqrt{2}}{2 \sqrt{R e \varepsilon}}+\frac{u_{0}}{2}\right) v+\frac{\sqrt{2} u}{2 \sqrt{R e \varepsilon}}+\frac{\bar{u}}{4 F r^{2} u_{0}} \\
u_{0} \zeta+\bar{u}
\end{array}\right) .
$$


In the sequel we use (3.22) rather than (3.21) and we drop the superscripts " $~ "$ ". Next, we remark that the transmission conditions $(3.20)(3.22)$ are a particular case of the generalized transmission conditions

$$
\begin{aligned}
\mathcal{B}_{-} X & =\frac{1}{R e} \partial_{x} U_{h}-\frac{u_{0}}{2} U_{h}+\frac{\alpha}{\sqrt{\varepsilon}} A U_{h}+\beta B \bar{U}_{h}, \\
\mathcal{B}_{+} X & ={ }^{t}\left(\begin{array}{c}
1 \\
-\frac{1}{R e} \partial_{x} U_{h}+\frac{u_{0}}{2} U_{h}+\frac{\alpha}{\sqrt{\varepsilon}} A U_{h}-\beta B \bar{U}_{h}, u_{0} \zeta+\bar{u}
\end{array}\right) \\
\text { with } \quad A & :=\left(\begin{array}{cc}
1 & -1 \\
1 & 1
\end{array}\right), \quad B:=\left(\begin{array}{cc}
1 & -1 / 2 \\
-1 / 2 & 0
\end{array}\right) .
\end{aligned}
$$

The original transmission operators (3.20)-(3.22) correspond to the choice

$$
\alpha=\alpha_{\text {Tay }}:=\frac{1}{\sqrt{2 R e}}, \quad \beta=\beta_{\text {Tay }}:=\frac{1}{2 F r^{2} u_{0}}
$$

where the subscript Tay means that these values have been obtained through Taylor expansion.

Notice that $\mathcal{B}_{-} X$ and $\left(\mathcal{B}_{+} X\right)_{(1,2)}$ do not depend on the water height $\zeta$, so we may rewrite $\mathcal{B}_{-} X=\mathcal{B}_{-}^{U_{h}} U_{h}$ and $\mathcal{B}_{+} X=\left(\mathcal{B}_{+}^{U_{h}} U_{h}, \mathcal{B}_{+}^{\zeta} X\right)^{t}$ as

$$
\mathcal{B}_{ \pm}^{U_{h}} U_{h}:=\mp \frac{1}{R e} \partial_{x} U_{h} \pm \frac{u_{0}}{2} U_{h}+\frac{\alpha}{\sqrt{\varepsilon}} A U_{h} \mp \beta B \bar{U}_{h} \quad \mathcal{B}_{+}^{\zeta} X:=u_{0} \zeta+\bar{u}
$$

Let us emphasize the identity:

$$
\mathcal{B}_{+}^{U_{h}} U_{h}+\mathcal{B}_{-}^{U_{h}} U_{h}=2 \frac{\alpha}{\sqrt{\varepsilon}} A U_{h} .
$$

This relation will be useful both for defining a weak formulation of the algorithm in Section 5 and for the numerical implementation of this algorithm in Section 6 . Finally the Schwarz waveform relaxation algorithm (3.1) is given by

$$
\left\{\begin{array} { c l r } 
{ \operatorname { P E } ( X _ { - } ^ { n + 1 } ) = 0 } & { \text { on } \Omega _ { T } ^ { - } , } \\
{ X _ { - } ^ { n + 1 } ( \cdot , 0 ) = X _ { i } } & { \text { on } \Omega ^ { - } , } \\
{ \mathcal { B } _ { - } ^ { U _ { h } } U _ { h , - } ^ { n + 1 } = \mathcal { B } _ { - } ^ { U _ { h } } U _ { h , + } ^ { n } } & { \text { on } \Gamma _ { T } , }
\end{array} \quad \left\{\begin{array}{ll}
\operatorname{PE}\left(X_{+}^{n+1}\right)=0 & \text { on } \Omega_{T}^{+}, \\
X_{+}^{n+1}(\cdot, 0)=X_{i} & \text { on } \Omega^{+}, \\
\mathcal{B}_{+}^{U_{h}} U_{h,+}^{n+1}=\mathcal{B}_{+}^{U_{h}} U_{h,-}^{n} & \text { on } \Gamma_{T}, \\
\mathcal{B}_{+}^{\zeta} X_{+}^{n+1}=\mathcal{B}_{+}^{\zeta} X_{-}^{n} & \text { on } \gamma_{T} .
\end{array}\right.\right.
$$

where the operators $\mathcal{B}_{ \pm}^{U_{h}}, \mathcal{B}_{+}^{\zeta}$ are defined by equalities $(3.25)(3.27)$ and where $\alpha$ and $\beta$ are free parameters.

These generalized transmission operators can now be optimized with respect to the two parameters $\alpha$ and $\beta$. We will present a numerical procedure in Section 6 .

4. Well-posedness of the linearized Primitive Equations. In the previous sections we have performed formal computations on the linearized Primitive Equations leading to the construction of the Schwarz waveform relaxation algorithm (3.29). The aim of this section is to be more precise: we will define a weak formulation of 
the system (2.8)-(2.12) and then prove that this system is well-posed in the natural spaces associated to this weak formulation.

From now on we relax the boundary condition on the bottom, i.e. we assume $\alpha_{b} \geq 0$ instead of $\alpha_{b}=0$. Moreover, in order to prepare the study of the well posedness of the algorithm (3.29) in the next section, we consider non-homogeneous right-hand sides $Y=\left(F_{1}, F_{2}, f\right)=Y(x, y, z, t)$. The system of linearized primitive equations $\operatorname{PE}(X)=Y$ is given by

$$
\begin{array}{rlrl}
\left\{\partial_{t}+U_{0} \cdot \nabla_{h}-\frac{1}{R e} \Delta_{h}-\frac{1}{R e^{\prime}} \partial_{z}^{2}+\frac{1}{\varepsilon} C\right\} U_{h}+\frac{1}{F r^{2}} \nabla_{h} \zeta=F & & \text { in } \Omega_{T}, \\
\partial_{z} U_{h}(x, y, 0, t) & =0 & & \text { on } \omega_{T}, \\
-\partial_{z} U_{h}(x, y,-1, t)+\alpha_{b} U_{h}(x, y,-1, t) & =0 & & \text { on } \omega_{T}, \\
\left\{\partial_{t}+U_{0} \cdot \nabla_{h}\right\} \zeta+\nabla_{h} \cdot \bar{U}_{h} & =f & & \text { in } \omega_{T} .
\end{array}
$$

We supplement this system with the initial conditions

$$
\begin{array}{cc}
U_{h}(\cdot, 0)=U_{h, i}, & \text { in } \Omega, \\
\zeta(\cdot, 0)=\zeta_{i} & \text { in } \omega .
\end{array}
$$

Note that, for the unknown $U_{h}$, system (4.1)-(4.2), (4.4) is a classical linear parabolic problem with a source term depending on $\zeta$. On the other hand, $\zeta$ solves the linear transport problem (4.3), (4.5) with a source term depending on $\bar{U}_{h}$.

We will proceed as follows: first we recall the classical weak formulations both for the parabolic problem (with prescribed water height) and for the transport equations (with prescribed velocity). These two problems define two maps $\mathcal{S}_{1}: \zeta \mapsto U_{h}$ and $\mathcal{S}_{2}: U_{h} \mapsto \zeta$. Finally we define the weak solutions of the Primitive Equations to be the fixed points of the map $\tau:\left(U_{h}, \zeta\right) \mapsto\left(\mathcal{S}_{1}(\zeta), \mathcal{S}_{2}\left(U_{h}\right)\right)$ and conclude by proving the existence of a unique fixed point.

Let us first introduce some functional spaces and some notations. We will work with initial conditions and right hand sides satisfying

$$
U_{h, i} \in H:=L^{2}\left(\Omega, \mathbf{R}^{2}\right), \quad \zeta_{i} \in L^{2}(\omega), \quad F \in L^{2}\left(0, T ; \mathcal{V}^{\prime}\right), \quad f \in L^{2}\left(\omega_{T}\right),
$$

where $\mathcal{V}^{\prime}$ is the topological dual of $\mathcal{V}:=H^{1}\left(\Omega, \mathbf{R}^{2}\right)$.

The weak solutions will satisfy

$U_{h} \in C([0, T], H) \cap L^{2}(0, T ; \mathcal{V}), \quad \zeta \in C\left([0, T], L^{2}(\omega)\right) \cap C\left(\mathbf{R}_{x}, L^{2}\left(\mathbf{R}_{y} \times(0, T)\right)\right)$.

We will need the following bilinear forms:

$$
\begin{aligned}
a(U, V):=\frac{1}{R e}\left(\nabla_{h} U, \nabla_{h} V\right)_{\Omega_{T}}+\frac{1}{R e^{\prime}}\left(\partial_{z} U, \partial_{z} V\right)_{\Omega_{T}}+\frac{\alpha_{b}}{R e^{\prime}}(U, V)_{\omega_{-1, T}} \\
+\frac{1}{\varepsilon}(C U, V)_{\Omega_{T}}+\left(U_{0} \cdot \nabla U, V\right)_{\Omega_{T}} \\
c(\zeta, V):=\frac{1}{F r^{2}}\left(\zeta e_{x}, \partial_{x} V\right)_{\Omega_{T}} .
\end{aligned}
$$

where $\omega_{-1}:=\mathbf{R}_{x} \times \mathbf{R}_{y} \times\{-1\}_{z}$ and $(U, V)_{\Sigma}$ denotes the $L^{2}$ scalar product on $\Sigma$. 
Assuming that we have a strong solution, and taking the scalar product of equation (4.1) with $V \in \mathcal{D}\left(\bar{\Omega} \times(0, T), \mathbf{R}^{2}\right)$, we obtain (after integrating by parts) the following weak formulation for the equations governing the horizontal velocities:

$$
\forall V \in \mathcal{D}\left(\bar{\Omega} \times(0, T), \mathbf{R}^{2}\right), \quad\left(\partial_{t} U_{h}, V\right)_{\Omega_{T}}+a\left(U_{h}, V\right)=c(\zeta, V)+\langle F, V\rangle .
$$

Definition 4.1. Let $F \in L^{2}\left(0, T ; \mathcal{V}^{\prime}\right)$ and $\zeta \in L^{2}\left(\omega_{T}\right)$, we say that $U_{h} \in$ $L^{2}(0, T ; \mathcal{V})$ is a weak solution of the system (4.1) if (4.6) holds.

Proposition 4.2. Let $U_{h, i} \in L^{2}(\Omega), F \in L^{2}\left(0, T ; \mathcal{V}^{\prime}\right)$ and $\zeta \in L^{2}\left(\omega_{T}\right)$, there exists a unique weak solution $U_{h} \in C([0, T] ; H) \cap L^{2}(0, T ; \mathcal{V})$ of $(4.1)$ satisfying the initial condition (4.4). Moreover, we have the energy inequality

$$
\begin{array}{r}
\frac{1}{2}\left\|U_{h}\right\|_{\Omega}^{2}(t)+\int_{0}^{t}\left\{\frac{1}{R e}\left\|\nabla_{h} U_{h}\right\|_{\Omega}^{2}(s)+\frac{1}{R e^{\prime}}\left\|\partial_{z} U_{h}\right\|_{\Omega}^{2}(s)+\frac{\alpha_{b}}{R e^{\prime}}\left\|U_{h}\right\|_{\omega_{-1}}^{2}(s)\right\} d s \\
\leq \frac{1}{2}\left\|U_{h, i}\right\|_{\Omega}^{2}+\int_{0}^{t}\left\{\left\langle F, U_{h}\right\rangle(s)+\left(\partial_{x} \bar{u}, \zeta\right)_{\omega}(s)\right\} d s
\end{array}
$$

Proof. The method is classical. We obtain the existence of a solution satisfying (4.7) by the Galerkin method.

Regularizing in time and using the weak formulation, we see that any solution satisfies (4.7) and uniqueness follows by the energy method.

Let us turn our attention to the equations (4.3), (4.5) governing the evolution of the water height $\zeta$. This is a linear transport equation with constant coefficients and a source term. Assuming that $\zeta$ is a strong solution, multiplying (4.3) by a test function $\chi \in \mathcal{D}(\omega \times[0, T))$, integrating on $\omega_{T}$, integrating by parts in space and time and then using the initial condition (4.5), we obtain

$$
\begin{aligned}
\forall \chi \in \mathcal{D}(\omega \times & {[0, T)) } \\
& -\left(\zeta,\left\{\partial_{t}+U_{0} \cdot \nabla_{h}\right\} \chi\right)_{\omega_{T}}=\left(\zeta_{i}, \chi(\cdot, 0)\right)_{\omega}+\left(f-\nabla_{h} \cdot \bar{U}_{h}, \chi\right)_{\omega_{T}} .
\end{aligned}
$$

Definition 4.3. Let $f \in L^{2}\left(\omega_{t}\right), U_{h} \in L^{2}(0, T ; \mathcal{V})$ and $\zeta_{i} \in L^{2}(\omega)$, we say that $\zeta \in L^{2}\left(\omega_{T}\right)$ is a weak solution of the system (4.3), (4.5) if (4.8) holds.

Remark that the test function does not necessarily vanish at time 0 and that the initial condition is prescribed by the weak formulation.

Proposition 4.4. Let $f \in L^{2}\left(\omega_{t}\right), U_{h} \in L^{2}(0, T ; \mathcal{V})$ and $\zeta_{i} \in L^{2}(\Omega)$. There exists a unique weak solution $\zeta \in L^{2}\left(\omega_{T}\right)$ of (4.3), (4.5). Moreover this solution is given by the characteristic formula:

$$
\zeta(x, y, t)=\zeta_{i}\left(x-u_{0} t, y-v_{0} t\right)+\int_{0}^{t}\left(f-\nabla_{h} \cdot \bar{U}_{h}\right)\left(x-u_{0} s, y-v_{0} s, t-s\right) d s .
$$

This solution lies in $C\left([0, T] ; L^{2}(\omega)\right) \cap C\left(\mathbf{R}_{x} ; L^{2}\left(\mathbf{R}_{y} \times(0, T)\right)\right)$ and satisfies the following estimates for every $t \in[0, T]$ and every $x \in \mathbf{R}$,

$$
\begin{aligned}
\|\zeta(\cdot, \cdot, t)\|_{\omega} & \leq\left\|\zeta_{i}\right\|_{\omega}+\int_{0}^{t}\left\|f-\nabla_{h} \cdot \bar{U}_{h}\right\|_{\omega}(s) d s \\
\|\zeta(x, \cdot, \cdot)\|_{\gamma_{t}} & \leq \frac{1}{u_{0}}\left(\left\|\zeta_{i}\right\|_{\omega}+\int_{0}^{t}\left\|f-\nabla_{h} \cdot \bar{U}_{h}\right\|_{\omega}(s) d s\right) .
\end{aligned}
$$


Proof. First, notice that the estimates (4.10) (4.11) are direct consequences of the characteristic formula (4.9).

Next, if the data $\nabla_{h} \bar{U}_{h}, f$ and $\zeta_{i}$ were sufficiently smooth then the function $\zeta$ given by formula (4.9) would solve (4.8). Finally, by using standard regularization arguments, we obtain the existence of a solution of (4.8) through formula (4.9).

We now prove uniqueness. By linearity we may assume that the data $\nabla_{h} \bar{U}_{h}, f$ and $\zeta_{i}$ vanish. Then let $\psi \in \mathcal{D}(\omega)$ and $\rho \in \mathcal{D}([0, T))$ and define the test function $\chi$ by $\chi(x, y, t):=-\psi\left(x-u_{0} t, y-v_{0} t\right) \int_{t}^{T} \rho(s) d s$, so that:

$$
\partial_{t} \chi+U_{0} \cdot \nabla \chi=\psi\left(x-u_{0} t, y-v_{0} t\right) \rho(t)
$$

and (4.8) yields

$$
0=\int_{\omega_{T}} \zeta(x, t) \rho(t) \psi\left(x-u_{0} t, y-v_{0} t\right)=\int_{\omega_{T}} \zeta\left(x+u_{0} t, y+v_{0} t, t\right) \rho(t) \psi(x, y) .
$$

Since this is true for every $(\psi, \rho) \in \mathcal{D}(\omega) \times \mathcal{D}([0, T))$, we have $\zeta \equiv 0$ on $\omega_{T}$. $\square$

Finally, we define the notion of weak solution for the linearized primitive equations.

Definition 4.5. Let $Y=(F, f) \in L^{2}\left(\Omega_{T}, \mathcal{V}^{\prime}\right) \times L^{2}\left(\omega_{T}\right)$ and $X_{i}=\left(U_{h, i}, \zeta_{i}\right) \in$ $L^{2}(\Omega) \times L^{2}(\omega)$. We say that $X=\left(U_{h}, \zeta\right) \in C(0, T ; H) \times L^{2}\left(\omega_{T}\right)$ is a weak solution of (4.1)-(4.5) if the weak formulations (4.6) and (4.8) hold and if $U_{h}(\cdot, 0)=U_{h, i}$.

We can now state an existence and uniqueness result for the weak solutions of the linearized primitive equations.

TheOrem 4.1. Let $Y=(F, f) \in L^{2}\left(\Omega_{T}, \mathcal{V}^{\prime}\right) \times L^{2}\left(\omega_{T}\right)$ and $X_{i}=\left(U_{h, i}, \zeta_{i}\right) \in$ $L^{2}(\Omega) \times L^{2}(\omega)$. There exists a unique weak solution $X=\left(U_{h}, \zeta\right) \in(C(0, T ; H) \cap$ $\left.L^{2}(0, T ; \mathcal{V})\right) \times L^{2}\left(\omega_{T}\right)$ of $(4.1)-(4.5)$.

Proof. The right hand side $Y$ and the initial condition $X_{i}$ being fixed, Proposition 4.2 and Proposition 4.4 define two maps

$$
S_{1}: L^{2}\left(\omega_{T}\right) \rightarrow C(0, T ; H) \cap L^{2}(0, T ; \mathcal{V}), \quad \zeta \mapsto U_{h},
$$

and

$$
S_{2}: L^{2}(0, T, \mathcal{V}) \rightarrow C\left([0, T] ; L^{2}(\omega)\right) \cap C\left(\mathbf{R}_{x} ; L^{2}(\mathbf{R} \times(0, T))\right), \quad U_{h} \mapsto \zeta
$$

Denoting by $\mathcal{T}$ the affine mapping $\left(U_{h}, \zeta\right) \mapsto\left(S_{1}(\zeta), S_{2}\left(U_{h}\right)\right)$, the function $X$ is a weak solution of (4.1)-(4.5) if and only if it is a fixed point of $\mathcal{T}$ in

$$
\mathcal{E}_{T}:=L^{2}(0, T ; \mathcal{V}) \times C\left([0, T], L^{2}(\omega)\right) .
$$

Let $X_{1}, X_{2} \in \mathcal{E}_{T}$ and let $\left(U_{h}, \zeta\right):=X_{1}-X_{2}$ and $\left(\tilde{U}_{h}, \tilde{\zeta}\right):=\mathcal{T}\left(X_{1}\right)-\mathcal{T}\left(X_{2}\right)$, by linearity, using (4.7), we get for $0 \leq t \leq T$,

$$
\begin{aligned}
\frac{1}{2}\left\|\tilde{U}_{h}\right\|_{\Omega}^{2}(t)+\int_{0}^{t}\left\{\frac{1}{R e}\left\|\nabla_{h} \tilde{U}_{h}\right\|_{\Omega}^{2}(s)\right. & \left.+\frac{1}{R e^{\prime}}\left\|\partial_{z} \tilde{U}_{h}\right\|_{\Omega}^{2}(s)\right\} d s \leq \int_{0}^{t}\left(\partial_{x} \overline{\tilde{u}}, \zeta\right)_{\omega}(s) d s \\
\leq & \left(\int_{0}^{t}\left\|\nabla \tilde{U}_{h}\right\|_{\Omega}^{2}(s) d s\right)^{1 / 2}\left(\int_{0}^{t}\|\zeta\|_{\omega}^{2}(s) d s\right)^{1 / 2}
\end{aligned}
$$


By Young's inequality, we may absorb the term in $\nabla \tilde{U}_{h}$ in the left hand side and get:

$$
\left\|\tilde{U}_{h}\right\|_{\Omega}^{2}(t)+\int_{0}^{t}\left\|\nabla \tilde{U}_{h}\right\|_{\Omega}^{2}(s) d s \leq \kappa t \sup _{s \in[0, t]}\left\{\|\zeta\|_{\omega}^{2}(s)\right\} \quad \text { for } 0 \leq t \leq T,
$$

for some $\kappa>0$. Now (4.10) and the Cauchy Schwarz inequality yield

$$
\|\tilde{\zeta}\|_{\omega}^{2}(t) \leq t \int_{0}^{t}\left\|\nabla U_{h}\right\|_{\Omega}^{2}(s) d s, \quad \text { for } 0 \leq t \leq T .
$$

Finally, inequalities (4.12) (4.13) imply that, for $T^{\prime} \in(0, T]$ small enough, the mapping $\mathcal{T}$ is strictly contracting in $\mathcal{E}_{T^{\prime}}$ yielding the existence of a unique fixed point of $\mathcal{T}$ in $\mathcal{E}_{T^{\prime}}$. Repeating the argument on the intervals $\left[T^{\prime}, 2 T^{\prime}\right],\left[2 T^{\prime}, 3 T^{\prime}\right], \ldots$ we obtain the result on $[0, T]$.

5. Weak formulation of the Schwarz waveform relaxation algorithm. In this section we establish that algorithm (3.29) is well defined. First, we will define weak formulations for the two sub-problems and prove that they are well-posed. We will pay a particular attention to the weak form of the transmission conditions. In particular we will establish that the solutions $X_{ \pm}^{n+1}$ of the $n^{\text {th }}$ step of the algorithm (3.29) are in the right spaces, allowing the construction of the transmission conditions for the next step.

As in the previous section, we also consider non-homogeneous right-hand sides $Y=$ $(F, f)$. Every step of the algorithm may be split in the two following sub-problems. First in the domain $\{x<0\}$, we search for a solution $X_{-}^{n+1}:=X_{-}=\left(U_{h,-}, \zeta_{-}\right)$ solving the initial and boundary value parabolic problem,

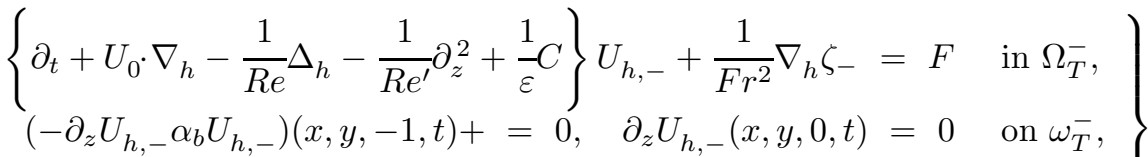

$$
\begin{aligned}
& \mathcal{B}_{-}^{U_{h}} U_{h,-}=\mathcal{B}_{-}^{U_{h}} U_{h,+}^{n} \quad \text { on } \Gamma_{T}, \quad U_{h,-}^{n+1}(\cdot, 0)=U_{h, i} \quad \text { in } \Omega^{-},
\end{aligned}
$$

and the transport problem,

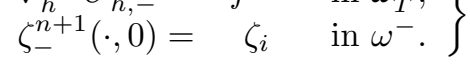

In the right subdomain $\{x>0\}$ we search for a solution $X_{+}^{n+1}:=X_{+}=\left(U_{h,+}, \zeta_{+}\right)$ solving the initial and boundary value parabolic problem,

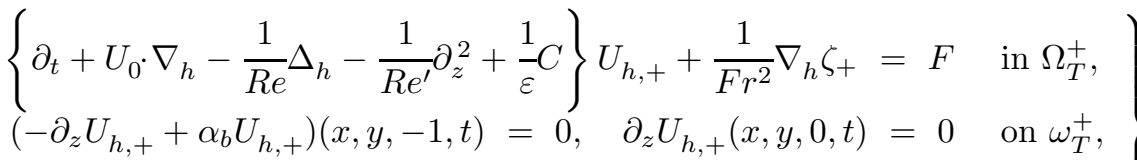

$$
\begin{aligned}
& \mathcal{B}_{+}^{U_{h}} U_{h,+}=\mathcal{B}_{+}^{U_{h}} U_{h,-}^{n} \quad \text { on } \Gamma_{T}, \quad U_{h,+}(\cdot, 0)=U_{h, i} \quad \text { in } \Omega^{+},
\end{aligned}
$$

and the transport problem with entering characteristics on the boundary $\gamma_{T}$,

$$
\begin{aligned}
& \left.\left\{\partial_{t}+U_{0} \cdot \nabla_{h}\right\} \zeta_{-}+\nabla_{h} \cdot \bar{U}_{h,+}=f \quad \text { in } \omega_{T}^{+},\right\} \\
& \left.\mathcal{B}_{+}^{\zeta} X_{+}=\mathcal{B}_{+}^{\zeta} X_{-}^{n} \quad \text { on } \gamma_{T}, \quad \zeta_{+}(\cdot, 0)=\zeta_{i} \quad \text { in } \omega^{+} .\right\}
\end{aligned}
$$


To prove that these two sub-problems are well-posed, we proceed as in Section 4. First we study the parabolic problems with prescribed water heights: we introduce a weak formulation for these problems and prove that they are well-posed. Then we study the transport equations, introduce their weak formulations and establish their well-posedness. Finally, the solutions of the coupled parabolic-transport problems are obtained via a fixed point method.

As in Section 4, the initial condition $X_{i}\left(U_{h, i}, \zeta_{i}\right)$ satisfy $U_{h, i} \in H, \zeta_{i} \in L^{2}(\omega)$. We choose right hand sides $Y=(F, f)$ in $L^{2}(0, T ; H) \times L^{2}\left(\omega_{t}\right)$. (In Section 4, we only assumed $F \in L^{2}\left(0, T ; \mathcal{V}^{\prime}\right)$, but here this choice would cause difficulties at the interface). We will search for weak solutions $X_{ \pm}=\left(U_{h, \pm}, \zeta_{ \pm}\right)$in the spaces,

$$
\begin{aligned}
U_{h, \pm} & \in C\left([0, T], H^{ \pm}\right) \quad \cap \quad L^{2}\left(0, T ; \mathcal{V}^{ \pm}\right), \\
\zeta_{ \pm} & \in C\left([0, T], L^{2}\left(\omega^{ \pm}\right)\right) \quad \cap \quad C\left(\mathbf{R}_{ \pm, x}, L^{2}\left(\mathbf{R}_{y} \times(0, T)_{t}\right)\right),
\end{aligned}
$$

with $H^{ \pm}:=L^{2}\left(\Omega^{ \pm}, \mathbf{R}^{2}\right)$ and $\mathcal{V}^{ \pm}:=H^{1}\left(\Omega^{ \pm}, \mathbf{R}^{2}\right)$.

For later use, we also introduce a space of functions defined on the interface $\Gamma$ :

$$
\mathcal{W}:=H^{1 / 2}\left(\Gamma, \mathbf{R}^{2}\right) .
$$

5.1. The parabolic problems. Let us define the weak-formulation for the parabolic problems (5.1) and (5.3). First we introduce the bilinear forms $a^{ \pm}$and $c^{ \pm}$:

$$
\begin{gathered}
a^{ \pm}(U, V):=\frac{1}{R e}\left(\nabla_{h} U, \nabla_{h} V\right)_{\Omega_{T}^{ \pm}}+\frac{1}{R e^{\prime}}\left(\partial_{z} U, \partial_{z} V\right)_{\Omega_{T}^{ \pm}}+\frac{\alpha_{b}}{R e^{\prime}}(U, V)_{\omega_{-1, T}^{ \pm}} \\
+\frac{1}{\varepsilon}(C U, V)_{\Omega_{T}^{ \pm}}+\left(U_{0} \cdot \nabla U, V\right)_{\Omega_{T}^{ \pm}}, \\
c^{ \pm}(\zeta, V)=\frac{1}{F r^{2}}\left(\zeta e_{x}, \partial_{x} \bar{V}\right)_{\omega_{T}^{ \pm}} \pm \frac{1}{F r^{2}}\left(\zeta e_{x}, \bar{V}\right)_{\gamma_{T}},
\end{gathered}
$$

where $\omega_{-1}^{ \pm}:=\mathbf{R}_{x}^{ \pm} \times \mathbf{R}_{y} \times\{-1\}_{z}$.

Next, taking the scalar product of the first equation of (5.1) or (5.3) with some test map $V \in \mathcal{D}\left(\overline{\Omega^{ \pm}} \times(0, T), \mathbf{R}^{2}\right)$, we obtain:

$$
\left(\partial_{t} U_{h, \pm}, V\right)_{\Omega^{ \pm} \times(0, T)}+a^{ \pm}\left(U_{h, \pm}, V\right)=c^{ \pm}\left(\zeta_{ \pm}, V\right) \mp \frac{1}{R e}\left(\partial_{x} U_{h, \pm}, V\right)_{\Gamma}+(F, V)_{\Omega_{T}^{ \pm}} .
$$

Then, using the transmission conditions to express $\partial_{x} U_{h, \pm}$ on $\Gamma$, we get

$$
\begin{aligned}
\left(\partial_{t} U_{h, \pm}, V\right)_{\Omega^{ \pm} \times(0, T)}+a^{ \pm}\left(U_{h, \pm}, V\right)+ & b^{ \pm}\left(U_{h, \pm}, V\right) \\
& =c^{ \pm}\left(\zeta_{ \pm}, V\right)+\left(\mathcal{B}_{ \pm}^{U_{h}} U_{h, \mp}^{n}, V\right)_{\Gamma}+(F, V)_{\Omega_{T}^{ \pm}}
\end{aligned}
$$

with

$$
b^{ \pm}(U, V):= \pm \frac{u_{0}}{2}(U, V)_{\Gamma}+\frac{\alpha}{\sqrt{\varepsilon}}(A U, V)_{\Gamma} \mp \beta(B \bar{U}, \bar{V})_{\Gamma}
$$

We are still not satisfied with this weak formulation. More precisely, the knowledge of $\partial_{x} U_{h, \mp}^{n}$ on the boundary $\Gamma \times(0, T)$ is needed for defining the term $\left(\mathcal{B}_{ \pm}^{U_{h}} U_{h, \mp}^{n}, V\right)_{\Gamma}$ in 
the right hand side. Unfortunately, (5.5) only gives: $\partial_{x} U_{h, \mp}^{n} \in L^{2}\left(\Omega^{ \pm} \times(0, T)\right)$ which is not sufficient to define a trace. To overcome this difficulty, we use relation (3.28) to define recursively the terms $\left(\mathcal{B}_{ \pm}^{U_{h}} U_{h, \mp}^{n}, V\right)_{\Gamma}$. Indeed, for strong solutions, we have on $\Gamma_{T}$

$$
\mathcal{B}_{\mp}^{U_{h}} U_{h, \pm} \stackrel{(3.28)}{=}-\mathcal{B}_{ \pm}^{U_{h}} U_{h, \pm}+2 \frac{\alpha}{\sqrt{\varepsilon}} A U_{h, \pm} \stackrel{(3.29)}{=}-\mathcal{B}_{ \pm}^{U_{h}} U_{h, \mp}^{n}+2 \frac{\alpha}{\sqrt{\varepsilon}} A U_{h, \pm} .
$$

Thus, identifying $\mathcal{B}_{ \pm}^{U_{h}} U_{h, \mp}^{n}$ with a distribution $\mathcal{B}_{ \pm}^{n} \in L^{2}\left(0, T ; \mathcal{W}^{\prime}\right)$, where $\mathcal{W}$ denotes the space $H^{1 / 2}\left(\Gamma, \mathbf{R}^{2}\right)$; we obtain a weak formulation of the algorithm for the horizontal velocities:

Definition 5.1. Assuming that the functions $\zeta_{ \pm}=\zeta_{ \pm}^{n+1}$ are known, the weak formulation of the parabolic part (5.1) and (5.3) of the algorithm (3.29) are defined as follows:

For the first step, we choose

$$
\mathcal{B}_{ \pm}^{0} \in L^{2}\left(0, T ; \mathcal{W}^{\prime}\right)
$$

Then for $n \geq 0$, the horizontal velocity is defined by $U_{h, \pm}^{n+1}=U_{h, \pm}$ where $U_{h, \pm}$ solves

$$
\begin{array}{r}
\forall V \in \mathcal{D}\left(\overline{\Omega^{ \pm}} \times(0, T), \mathbf{R}^{2}\right), \quad\left(\partial_{t} U_{h, \pm}, V\right)_{\Omega^{ \pm} \times(0, T)}+a^{ \pm}\left(U_{h, \pm}, V\right)+b^{ \pm}\left(U_{h, \pm}, V\right) \\
=c^{ \pm}\left(\zeta_{ \pm}, V\right)+\left\langle\mathcal{B}_{ \pm}^{n}, V\right\rangle_{\Gamma_{T}}+(F, V)_{\Omega_{T}^{ \pm}},
\end{array}
$$

where $a^{ \pm}, c^{ \pm}$, and $b^{ \pm}$are defined in (5.7)-(5.9). Once $U_{h, \pm}^{n+1}$ is known, we can define the boundary conditions for the next step in the opposite domain by

$$
\mathcal{B}_{\mp}^{n+1}:=-\mathcal{B}_{ \pm}^{n}+2 \frac{\alpha}{\sqrt{\varepsilon}} A U_{h, \pm \mid \Gamma_{T}}^{n+1} .
$$

Notice that assuming that the maps $U_{h, \pm}^{n+1}$ satisfy (5.5) then their traces on $\Gamma_{T}$ are well defined in $L^{2}(0, T ; \mathcal{W}) \subset L^{2}\left(0, T ; \mathcal{W}^{\prime}\right)$. Consequently, the transmission conditions $\mathcal{B}_{\mp}^{n+1}$ defined recursively by (5.11) stay in the space $L^{2}\left(0, T ; \mathcal{W}^{\prime}\right)$.

Proposition 5.2. Let $U_{h, i} \in H, F \in L^{2}(0, T ; H), \mathcal{B}_{ \pm}^{n} \in L^{2}\left(0, T ; \mathcal{W}_{ \pm}^{\prime}\right)$ and $\zeta_{ \pm}(=$ $\left.\zeta_{ \pm}^{n+1}\right)$ satisfying (5.6). Then there exists a unique $U_{h, \pm}^{n+1}=U_{h, \pm}$ with regularity (5.5) satisfying (5.10) and the initial condition $U_{h, \pm}^{n+1}(0) \equiv U_{h, i}$ on $\Omega_{ \pm}$. Moreover, we have the energy inequality

$$
\begin{gathered}
\frac{1}{2}\left\|U_{h, \pm}\right\|_{\Omega^{ \pm}}^{2}(t)+\left(\frac{\alpha}{\sqrt{\varepsilon}} \pm u_{0} / 2\right)\left\|U_{h, \pm}\right\|_{\Gamma_{t}}^{2} \mp \beta\left(\left\|\bar{u}_{ \pm}\right\|_{\gamma_{t}}^{2}-\left(\bar{u}_{ \pm}, \bar{v}_{ \pm}\right)_{\gamma_{t}}\right) \\
+\int_{0}^{t}\left\{\frac{1}{R e}\left\|\nabla_{h} U_{h, \pm}\right\|_{\Omega^{ \pm}}^{2}(s)+\frac{1}{R e^{\prime}}\left\|\partial_{z} U_{h, \pm}\right\|_{\Omega^{ \pm}}^{2}(s)+\frac{\alpha_{b}}{R e^{\prime}}\left\|U_{h, \pm}\right\|_{\omega_{-1}^{ \pm}}^{2}(s)\right\} d s \\
\leq \frac{1}{2}\left\|U_{h, i}\right\|_{\Omega^{ \pm}}^{2}+\left(F, U_{h, \pm}\right)_{\Omega_{t}^{ \pm}}+\left\langle\mathcal{B}_{ \pm}^{n}, U_{h, \pm}\right\rangle_{\Gamma_{t}} \\
+\int_{0}^{t}\left\{\left(\partial_{x} \bar{u}_{ \pm}, \zeta_{ \pm}\right)_{\omega^{ \pm}}(s) \pm\left(\bar{u}_{ \pm}, \zeta_{ \pm}\right)_{\gamma^{ \pm}}(s)\right\} d s .
\end{gathered}
$$


Proof. We proceed as in the proof of Proposition 4.2: we apply the Galerkin method. Here we only check that the a priori inequality (5.12) is sufficient for applying this method. In order to bound the quadratic terms in the left hand side of (5.12) and the last term in the right hand side, we will use the inequality

$$
\|U\|_{\Gamma}^{2} \leq 2\|U\|_{\Omega^{ \pm}}\left\|\partial_{x} U\right\|_{\Omega_{ \pm}}
$$

valid for $U \in \mathcal{V}_{ \pm}$. (To prove it, write $|U(0, y, z)|^{2}=2 \int_{-\infty}^{0}\left(\partial_{x} U \cdot U\right)\left(x^{\prime}, y, z\right) d x^{\prime}$ integrate on $\mathbf{R}_{y} \times(-1,0) z$ and use the Cauchy-Schwarz inequality). From this inequality, the Cauchy-Schwarz inequality, the Young inequality and the fact that the trace on $\Gamma$ defines a continuous embedding $\Pi: \mathcal{V}^{ \pm} \rightarrow \mathcal{W}$, we see that (5.12) implies

$$
\begin{aligned}
& \left\|U_{h, \pm}\right\|_{\Omega^{ \pm}}^{2}(t)+\int_{0}^{t}\left\|\nabla U_{h, \pm}\right\|_{\Omega^{ \pm}}^{2}(s) d s-\kappa \int_{0}^{t}\left\|U_{h, \pm}\right\|_{\Omega^{ \pm}}^{2}(s) d s \\
& \quad \leq \kappa\left\{\left\|U_{h, i}\right\|_{\Omega^{ \pm}}^{2}+\|F\|_{\Omega_{t}^{ \pm}}^{2}+\int_{0}^{t}\left\|\mathcal{B}_{ \pm}^{n}\right\|_{\mathcal{W}^{\prime}}^{2}(s) d s+\left\|\zeta_{ \pm}\right\|_{\Omega_{t}}^{2}+\left\|\zeta_{ \pm}\right\|_{\gamma_{t}}^{2}\right\}
\end{aligned}
$$

for some $\kappa>0$. This is sufficient to apply the Galerkin method.

The uniqueness follows from (5.13) and Gronwall Lemma.

5.2. The transport equations. We now consider that the velocities $U_{h, \pm}=$ $U_{h, \pm}^{n+1}$ are known and study the transport problems (5.2) and (5.4). We begin with the domain $\{x<0\}$. Proceeding exactly as in Section 4 , we obtain that a strong solution of problem (5.2) satisfies

$$
\begin{aligned}
\forall \chi \in \mathcal{D} & \left(\omega^{-} \times[0, T)\right), \\
& -\left(\zeta_{-},\left\{\partial_{t}+U_{0} \cdot \nabla_{h}\right\} \chi\right)_{\omega_{T}^{-}}=\left(\zeta_{i}, \chi(\cdot, 0)\right)_{\omega^{-}}+\left(f-\nabla_{h} \cdot \bar{U}_{h,-}, \chi\right)_{\omega_{T}^{-}}
\end{aligned}
$$

Definition 5.3. Let $f \in L^{2}\left(\omega_{t}\right), U_{h,-}\left(=U_{h,-}^{n+1}\right) \in L^{2}\left(0, T ; \mathcal{V}^{-}\right)$and $\zeta_{i} \in L^{2}(\omega)$. We say that $\zeta_{-} \in L^{2}\left(\omega_{T}^{-}\right)$is a weak solution of problem (5.2) if (5.14) holds.

The following result is proved exactly as Proposition 4.4

Proposition 5.4. Let $f \in L^{2}\left(\omega_{t}\right), U_{h,-}^{n+1} \in L^{2}\left(0, T ; \mathcal{V}^{-}\right)$and $\zeta_{i} \in L^{2}(\omega)$. There exists a unique weak solution $\zeta_{-}^{n+1}=\zeta_{-} \in L^{2}\left(\omega_{T}^{-}\right)$of (5.2). Moreover this solution is explicitly given by the formula:

$$
\zeta_{-}(x, y, t)=\zeta_{i}\left(x-u_{0} t, y-v_{0} t\right)+\int_{0}^{t}\left(f-\nabla_{h} \cdot \bar{U}_{h,-}\right)\left(x-u_{0} s, y-v_{0} s, t-s\right) d s .
$$

It belongs to $C\left([0, T] ; L^{2}\left(\omega^{-}\right)\right) \cap C\left((-\infty, 0]_{x} ; L^{2}\left(\mathbf{R}_{y} \times(0, T)\right)\right)$ and satisfies the following estimates for every $t \in[0, T]$ and every $x \leq 0$,

$$
\begin{aligned}
& \left\|\zeta_{-}(\cdot, t)\right\|_{\omega^{-}} \leq\left\|\zeta_{i}\right\|_{\omega^{-}}+\int_{0}^{t}\left\|f-\nabla_{h} \cdot \bar{U}_{h,-}\right\|_{\omega^{-}}(s) d s \\
& \left\|\zeta_{-}(x, \cdot)\right\|_{\gamma_{t}} \leq \frac{1}{u_{0}}\left(\left\|\zeta_{i}\right\|_{\omega^{-}}+\int_{0}^{t}\left\|f-\nabla_{h} \cdot \bar{U}_{h,-}\right\|_{\omega^{-}}(s) d s\right) .
\end{aligned}
$$

Once the solutions of (5.1)-(5.2) are known it is possible to define the transmission conditions on the water-height for the next step (see (3.27))

$$
\left.\mathcal{B}_{+}^{\zeta} X_{-}^{n+1}:=u_{0} \zeta_{-}^{n+1}(0, \cdot)\right)+\bar{u}_{-}^{n+1}(0, \cdot) .
$$


In the domain $x>0$, the situation is slightly different since there are ingoing characteristics on $\gamma_{T}$. So we choose test functions that do not necessarily vanish on the boundary and use the transmission condition to prescribe the value of the solution on $\gamma_{T}$. Finally, a solution of (5.4) satisfies

$$
\begin{aligned}
\forall \chi \in \mathcal{D}\left(\bar{\omega}^{+} \times[0, T)\right), \quad-\left(\zeta_{+},\left\{\partial_{t}+U_{0} \cdot \nabla_{h}\right\} \chi\right)_{\omega_{T}^{+}} \\
=\left(\zeta_{i}, \chi(\cdot, 0)\right)_{\omega^{+}}+\left(\zeta_{b}, \chi(0, \cdot)\right)_{\mathbf{R}_{t}}+\left(f-\nabla_{h} \cdot \bar{U}_{h,+}, \chi\right)_{\omega_{T}^{+}},
\end{aligned}
$$

where the boundary value $\zeta_{b}$ is defined on $\gamma_{T}$ by

$$
\zeta_{b}:=\frac{1}{u_{0}}\left\{\mathcal{B}_{+}^{\zeta} X_{-}^{n}-\bar{u}_{+}\right\} .
$$

Definition 5.5. Let $f \in L^{2}\left(\omega_{t}\right), U_{h,+}\left(=U_{h,+}^{n+1}\right) \in L^{2}\left(0, T ; \mathcal{V}^{+}\right), \zeta_{i} \in L^{2}(\omega)$. Assuming that $\zeta_{b}$ defined by (5.19) belongs to $L^{2}\left(\gamma_{T}\right)$, we say that $\zeta_{+}^{n+1}=\zeta_{+} \epsilon$ $L^{2}\left(\omega_{T}^{+}\right)$is a weak solution of problem (5.4) if (5.18) holds.

Using the characteristic method, we have

Proposition 5.6. Let $f, U_{h,+}\left(=U_{h,+}^{n+1}\right), \zeta_{i}$ and $\zeta_{b}$ be as in Definition 5.5. There exists a unique weak solution $\zeta_{+}^{n+1} \in L^{2}\left(\omega_{T}^{+}\right)$of (5.4). Moreover it is given by the characteristic formula:

$$
\zeta_{+}(x, y, t)=\zeta_{i}\left(x-u_{0} t, y-v_{0} t\right)+\int_{0}^{t}\left(f-\nabla_{h} \cdot \bar{U}_{h,+}\right)\left(x-u_{0} s, y-v_{0} s, t-s\right) d s
$$

if $x>u_{0} t$, and

$\zeta_{+}(x, y, t)=\zeta_{b}\left(y-\frac{v_{0}}{u_{0}} x, t-\frac{x}{u_{0}}\right)+\int_{0}^{\frac{x}{u_{0}}}\left(f-\nabla_{h} \cdot \bar{U}_{h,+}\right)\left(x-u_{0} s, y-v_{0} s, t-s\right) d s$, with $\zeta_{b}$ given by (5.19), if $x \leq u_{0} t$.

The solution belongs to $C\left([0, T] ; L^{2}\left(\omega^{+}\right)\right) \cap C\left([0,+\infty)_{x} ; L^{2}\left(\mathbf{R}_{y} \times(0, T)\right)\right)$ and satisfies the following estimates for every $t \in[0, T]$ and every $x \geq 0$,

$$
\begin{aligned}
\left\|\zeta_{+}(\cdot, t)\right\|_{\omega+} & \leq\left\|\zeta_{i}\right\|_{\omega+}+u_{0}\left\|\zeta_{b}\right\|_{\gamma_{t}}+\int_{0}^{t}\left\|f-\nabla_{h} \cdot \bar{U}_{h,+}\right\|_{\omega+}(s) d s, \\
\left\|\zeta_{+}(x, \cdot)\right\|_{\gamma_{t}} & \leq \frac{1}{u_{0}}\left(\left\|\zeta_{i}\right\|_{\omega^{+}}+u_{0}\left\|\zeta_{b}\right\|_{\gamma_{t}}+\int_{0}^{t}\left\|f-\nabla_{h} \cdot \bar{U}_{h,+}\right\|_{\omega+}(s) d s\right) .
\end{aligned}
$$

The needed number of iterations also depends on the length of the time domain. In practice, the interval $(0, T)$ is split in $P$ time windows of lengths $T / P$. The $S W R$ algorithm is run on each time window successively by using the solution at final time of the time window $p$ as initial data in time window $p+1$.

5.3. The algorithm is well defined. We will say that the algorithm is welldefined if starting from a reasonable initial guess, we are able to construct recursively the sequences $\left(X_{ \pm}^{n}\right)_{n>0}$. The rigorous definition of our algorithm is given below.

First we define a weak formulation for the left and right sub-problems at step $n$ of the algorithm.

Definition 5.7. Let $Y=(F, f) \in L^{2}\left(\Omega_{T}\right) \times L^{2}\left(\omega_{T}\right)$, let $X_{i}=\left(U_{h, i}, \zeta_{i}\right) \in$ $L^{2}(\Omega) \times L^{2}(\omega)$. For $n \geq 0$. 
- Let $\mathcal{B}_{-}^{n} \in L^{2}\left(0, T ; \mathcal{W}^{\prime}\right)$. Then $X_{-}^{n+1}=\left(U_{h,-}, \zeta_{-}\right)$is a weak solution of problem (5.1), (5.2) if it has regularity (5.5)-(5.6) and if $U_{h,-}$ (respectively $\left.\zeta_{-}\right)$is a weak solution of $(5.1)$ (respectively (5.2)).

- Let $\mathcal{B}_{+}^{n} \in L^{2}\left(0, T ; \mathcal{W}^{\prime}\right)$ and $\mathcal{B}_{+}^{\zeta} X_{-}^{n} \in L^{2}\left(\gamma_{T}\right)$. Then $X_{+}^{n+1}=\left(U_{h,+}, \zeta_{+}\right)$is a weak solution of problem (5.3), (5.4) if it has regularity (5.5)-(5.6) and if $U_{h,+}$, (respectively $\zeta_{+}$) is a weak solution of (5.3)(respectively (5.4)).

Then we give a weak formulation for the complete algorithm.

Definition 5.8. The weak formulation of Algorithm (3.29) is defined by

- Choose $\mathcal{B}_{+}^{\zeta} X_{-}^{0} \in L^{2}\left(\gamma_{T}\right)$ and $\mathcal{B}_{ \pm}^{0} \in L^{2}\left(0, T ; \mathcal{W}^{\prime}\right)$.

Then, for $n \geq 0$,

- Find $X_{-}^{n+1}$ weak solution of (5.1)-(5.2) and $X_{+}^{n+1}$ weak solution of (5.3)(5.4).

- Define the transmission conditions for step $n+1$ by (5.11) and (5.17).

THEOREM 5.1. With the hypotheses of Definition 5.7, there exists a unique weak solution $X_{-}^{n+1}$ (respectively $X_{+}^{n+1}$ ) of problem (5.1),(5.2) (respectively (5.3),(5.4)).

Proof. We only prove the result for the left sub-problem, the other one being similar. As in the proof of Theorem 4.1, we use a fixed point method. Let us introduce the spaces

$$
\begin{aligned}
& \mathcal{E}_{T}^{1}:=C\left([0, T], H^{-}\right) \quad \cap \quad L^{2}\left(0, T ; \mathcal{V}^{-}\right) \\
& \mathcal{E}_{T}^{2}:=C\left([0, T], L^{2}\left(\omega^{-}\right)\right) \quad \cap \quad C\left((-\infty, 0]_{x}, L^{2}\left(\mathbf{R}_{y} \times(0, T)_{t}\right)\right) .
\end{aligned}
$$

Proposition 5.2 and Proposition 5.4 define two affine mappings $\mathcal{S}_{1}: \mathcal{E}_{T}^{2} \rightarrow \mathcal{E}_{T}^{1}$, $\zeta_{-} \mapsto U_{h,-}$ and $\mathcal{S}_{2}: \mathcal{E}_{T}^{1} \rightarrow \mathcal{E}_{T}^{2}, U_{h,-} \mapsto \zeta_{-}$.

Setting $\mathcal{E}_{T}^{-}:=\mathcal{E}_{T}^{1} \times \mathcal{E}_{T}^{2}$, a function $X_{-}^{n+1}$ is a weak solution of problem (5.1),(5.2) if and only if it is a fixed point in $\mathcal{E}_{T}^{-}$of the mapping

$$
\mathcal{T}^{-}:\left(U_{h,-}, \zeta_{-}\right) \mapsto\left(\mathcal{S}_{1}^{-}\left(\zeta_{-}\right), \mathcal{S}_{2}^{-}\left(U_{h,-}\right)\right)
$$

We now show that $\mathcal{T}^{-}$has a unique fixed point. Let $X_{1}, X_{2} \in \mathcal{E}_{T}^{-}$and let $\left(U_{h,-}, \zeta_{-}\right):=$ $X_{1}-X_{2}$ and $\left(\tilde{U}_{h}, \tilde{\zeta}\right):=\mathcal{T}^{-}\left(X_{1}\right)-\mathcal{T}^{-}\left(X_{2}\right)$. By linearity $(5.13)$ yields

$\left\|\tilde{U}_{h,-}\right\|_{\Omega^{-}}^{2}(t)+\int_{0}^{t}\left\|\nabla \tilde{U}_{h,-}\right\|_{\Omega^{-}}^{2}(s) d s-\kappa \int_{0}^{t}\left\|\tilde{U}_{h,-}\right\|_{\Omega^{-}}^{2}(s) d s \leq \kappa\left\{\left\|\zeta_{-}\right\|_{\Omega_{t}}^{2}+\left\|\zeta_{-}\right\|_{\gamma_{t}}^{2}\right\}$,

for $0 \leq t \leq T$. And from Gronwall lemma, we obtain for $0 \leq t \leq T$,

$$
\begin{aligned}
\left\|\tilde{U}_{h,-}\right\|_{\Omega}^{2}(t)+\int_{0}^{t}\left\|\nabla \tilde{U}_{h,-}\right\|_{\Omega}^{2}(s) d s & \\
& \leq \kappa e^{\kappa T}\left\{t \sup _{[0, t]}\left\|\zeta_{-}(\cdot, s)\right\|_{\omega}^{2}+\sup _{\mathbf{R}_{-}}\left\|\zeta_{-}(w, \cdot)\right\|_{\gamma_{t}}^{2}\right\} .
\end{aligned}
$$

Now from (5.15) and (5.16), we get

$$
\left\|\tilde{\zeta_{-}}\right\|_{\omega^{-}}^{2}(t)+u_{0}\left\|\tilde{\zeta_{-}}(x, \cdot)\right\|_{\gamma_{t}} \leq t\left\|\nabla U_{h,-}\right\|_{\Omega_{t}^{-}}^{2} \quad \text { for } 0 \leq t \leq T
$$


Finally, we endow $\mathcal{E}_{t}^{-}$with the norm

$$
\begin{aligned}
& \left\|\left(U_{h,-}, \zeta_{-}\right)\right\|_{\mathcal{E}_{t}^{-}}:= \\
& \left(\sup _{[0, t]}\left\|U_{h,-}(\cdot, s)\right\|_{\Omega^{-}}^{2}+\left\|\nabla U_{h,-}\right\|_{\Omega_{t}^{-}}^{2}+\sup _{[0, t]}\left\|\zeta_{-}(\cdot, s)\right\|_{\omega}^{2}+2 \kappa e^{\kappa T} \sup _{\mathbf{R}_{-}}\left\|\zeta_{-}(x, \cdot)\right\|_{\gamma_{t}}^{2}\right)^{1 / 2}
\end{aligned}
$$

With this norm (5.20) (5.21) imply that for $T^{\prime} \in(0, T]$ small enough, $\mathcal{T}^{-}$is contracting in $\mathcal{E}_{T^{\prime}}$. This yields the existence of a unique fixed point of $\mathcal{T}^{-}$in $\mathcal{E}_{T^{\prime}}^{-}$. We obtain the result on $[0, T]$ by continuation.

Finally, we can state

THEOREM 5.2. The algorithm 5.8 is well-defined.

Proof. We have to check that for each step the hypotheses of Theorem 5.1 are satisfied. Since at step $n$, the solutions $X_{ \pm}^{n+1}$ have regularity $(5.5)(5.6)$, we deduce that $\mathcal{B}_{ \pm}^{n+1}$ defined by $(5.11)$ belongs to $L^{2}\left(0, T ; \mathcal{W}^{\prime}\right)$ and $\mathcal{B}_{+}^{\zeta} X_{-}^{n+1}$ defined by $(5.17)$ belongs to $L^{2}\left(\gamma_{T}\right)$. Thus the hypotheses of Theorem 5.1 hold for step $n+1$.

5.4. Convergence of the algorithm. Although we do not exhibit a proof here, we are able to establish the convergence of the algorithm in some cases. More precisely, if the matrices $A$ and $B$ defined by (3.25) are replaced by diagonal matrices $\tilde{A}$ and $\tilde{B}$, $\tilde{A}$ being positive definite and $\tilde{B}$ being non negative, then the algorithm converges. The proof relies on the energy method developed for the Shallow water equations without advection term in [23]. Modifying slightly the proof, we can allow $\tilde{A}$ and $\tilde{B}$ to have non vanishing skew-symmetric off-diagonal parts. This generalization still does not cover the situation (3.25). Nevertheless, numerical evidences of the convergence of the algorithm are given in the next section.

6. Numerical results. For the numerical applications we consider for simplicity a 2 dimensional domain and the related two dimensional $(x, z)$ version of the primitive equations (2.8)-(2.12). Note that the transmission conditions (3.23)-(3.24) are independent of the transverse $y$-variable and are not affected by this simplification.

Of course we also have to consider finite domains. So in this section we set

$$
\Omega:=(-L, L)_{x} \times(-H, 0)_{z}, \quad \omega:=(-L, L)_{x},
$$

where $H, L>0$ are the depth and length of the domain respectively. In the numerical tests, we impose homogeneous Dirichlet boundary conditions for the velocity $U_{h}=$ $(u, v)$ on the left and right boundaries of $\Omega$ and for the water height $\zeta$ on the left boundary.

We have performed numerical simulation in oder to assess the transmissions conditions (3.27). We have built these conditions at the continuous level with no link to a particular discretization. Therefore, we are free to choose any kind of discretization with any valid numerical scheme. In particular it is possible to consider non-matching grids by using interpolation and projection operators between grids (see [15]). However, in the next subsection we briefly describe the discretization of (3.29) we have used in practice. In particular, we will give some details on the discretization of the transmission conditions. 
6.1. Numerical scheme in the subdomains. Since the processes are the same in both subdomains $\Omega^{ \pm}$, we restrict ourselves to the subdomain $\Omega^{+}:=(0, L)_{x} \times$ $(-H, 0)_{z}$.

For the space discretization, we consider a regular cartesian grid and we apply a finite volume method. We introduce the horizontal space step $\Delta x$ and the vertical space step $\Delta z$. For Euler or Navier-Stokes type problems it is well known that a good way to recover some numerical stability is to compute velocities and pressure on different cells (see for instance [1] and the publications devoted to the so-called C-grids). Here we only deal with the horizontal velocity and the water height (only depending on $x$ and $t$ ) plays the role of the pressure. We thus have to introduce two types of finite volume meshes.

The first one is a $2 \mathrm{~d}$ finite volume mesh and is related to the computation of the velocities. Its cells are denoted $C_{I}:=\left\{X_{I}^{+}+(-\Delta x / 2, \Delta x / 2) \times(-\Delta z / 2, \Delta z / 2)\right\} \cap$ $\Omega_{+}$, where $X_{I}=\left(x_{i}, z_{j}\right):=(0,-H)+(i \Delta x, j \Delta z)$ for $I=(i, j) \in \mathbf{N}^{2}$.

The second grid is a $1 \mathrm{~d}$ finite volume mesh devoted to the computation of the water height. The cells of this second mesh will be denoted $c_{i+1 / 2}=\left\{x_{i+1 / 2}+\right.$ $(-\Delta x / 2, \Delta x / 2)\} \cap(0, L)$. where $x_{i+1 / 2}:=(i+1 / 2) \Delta x$. For the time discretization, we fix $\Delta t>0$ and note $t^{k}:=k \Delta t$ for $k \geq 0$.

We use a finite volume method so the discrete unknown represent mean values of the exact solution:

$$
u_{I, k}^{+, n} \simeq f_{C_{I}} u_{+}^{n}\left(\cdot, t_{k}\right), \quad v_{I, k}^{+, n} \simeq f_{C_{I}} v_{+}^{n}\left(\cdot, t_{k}\right), \quad \zeta_{i+1 / 2, k}^{+, n} \simeq f_{c_{i+1 / 2}} \zeta_{+}^{n}\left(\cdot, t_{k}\right) .
$$

We also need to define discrete quantities for the non-classical boundary conditions

$$
B_{j, k}^{U_{h}, n} \simeq f_{\left(z_{j}-\frac{\Delta z}{2}, z_{j}+\frac{\Delta z}{2}\right)} \mathcal{B}_{+}^{U_{h}} U_{h,-}^{n}\left(0, z, t_{k}\right) d z, \quad B_{k}^{\zeta,+, n} \simeq \mathcal{B}_{+}^{\zeta} X_{-}^{n}\left(0, t_{k}\right)
$$

The scheme is as follows, at every time step we solve the transport equation by the explicit upwind Finite Volume scheme, namely

$$
\zeta_{i+1 / 2, k+1}^{+, n+1}=\left(1-\frac{\Delta t}{\Delta x} u_{0}\right) \zeta_{i+1 / 2, k}^{+, n+1}+\frac{\Delta t}{\Delta x} u_{0} \zeta_{i-1 / 2, k}^{+, n+1}-\frac{\Delta t}{\Delta x}\left(\bar{u}_{i, k}^{+, n+1}-\bar{u}_{i-1, k}^{+, n+1}\right)
$$

This formula is only valid for $i \geq 1$. For $i=0$ the left boundary of the current cell $c_{0}$ belongs to the artificial boundary and the quantity $u_{0} \zeta_{-1 / 2, k}^{+, n+1}+\bar{u}_{-1, k}^{+, n+1}$ is not defined but thanks to (3.27), this is exactly the quantity given by the transmission operator, so at this point the scheme reads

$$
\zeta_{1 / 2, k+1}^{+, n+1}=\left(1-\frac{\Delta t}{\Delta x} u_{0}\right) \zeta_{1 / 2, k}^{+, n+1}-\frac{\Delta t}{\Delta x} \bar{u}_{1, k}^{+, n+1}+\frac{\Delta t}{\Delta x} B_{k}^{\zeta,+, n},
$$

where $B_{k}^{\zeta,+, n}=u_{0} \zeta_{-1 / 2, k}^{-, n}+\bar{u}_{-1, k}^{-, n}$ has been computed at the previous step.

Next we use the standard Finite Volume procedure for discretizing the momentum equations. Namely, we integrate the momentum equations on the space time cell $C_{I} \times\left[t_{k}, t_{k+1}\right]$, we integrate by parts the terms involving time or space derivatives. and obtain that the variation of $U_{h}$ between two time steps is given by a sum of fluxes through the boundary $\partial C_{I} \times\left[t_{k}, t_{k+1}\right]$. To compute these fluxes, we use approximations (6.1) and centered finite difference formulas (for the derivatives). 
For every cell such that $\overline{C_{I}} \subset \Omega_{+}$, we recover the well-known Crank-Nicolson scheme. It is known to be second order accurate and unconditionally stable.

For the physical boundary conditions (2.9) we use the ghost cells method to compute the fluxes through the physical boundary.

Finally, if $C_{I}$ is a cell with a boundary intersecting the axis $\{x=0\}$, (i.e. $i=0$, $\left.C_{I}=(0, \Delta x / 2) \times\left(z_{j}-\Delta z / 2, z_{j}+\Delta z / 2\right)\right)$, we have to discretize the flux through the artificial boundary. More precisely we have to discretize the quantities

$$
q_{j, k}^{n+1}:=f_{\left(z_{j}-\frac{\Delta z}{2}, z_{j}+\frac{\Delta z}{2}\right)}\left(\frac{1}{R e} \partial_{x} U_{h,+}^{n+1}-\frac{\zeta_{+}^{n+1}}{F r^{2}}\right)\left(0, z, t_{k}\right) d z .
$$

First, from the definition (3.27) of the transmission operators, we have

$$
\begin{aligned}
\zeta_{+}^{n+1}\left(0, t_{k}\right) & =\mathcal{B}_{+}^{\zeta} X_{+}^{n+1}\left(0, t_{k}\right)-\frac{\bar{u}_{+}^{n+1}}{u_{0}}\left(0, t_{k}\right), \\
\frac{1}{R e} \partial_{x} U_{h,+}^{n+1} & =\left\{-\mathcal{B}_{+}^{U_{h}} U_{h,+}^{n+1}+\frac{u_{0}}{2} U_{h,+}^{n+1}+\frac{\alpha}{\sqrt{\varepsilon}} A U_{h,+}^{n+1} \mp \beta B \bar{U}_{h,+}^{n+1}\right\}\left(0, z, t_{k}\right)
\end{aligned}
$$

Using the transmission conditions $\mathcal{B}_{+}^{U_{h}} U_{h,+}^{n+1}=\mathcal{B}_{+}^{U_{h}} U_{h,-}^{n}$ and $\mathcal{B}_{+}^{\zeta} X_{+}^{n+1}=\mathcal{B}_{+}^{\zeta} X_{-}^{n}$ on the artificial boundary and then using the discretizations (6.1)(6.2), we obtain

$$
q_{j, k}^{n+1} \simeq-B_{j, k}^{U_{h},+, n}+\left(\frac{u_{0}}{2}+\frac{\alpha}{\sqrt{\varepsilon}} A-\beta B\right) U_{h, 0, j, k}^{+, n+1}-\frac{1}{F r^{2}} B_{k}^{\zeta,+, n}+\frac{1}{F r^{2}} \frac{\bar{u}_{0, j, k}^{+, n+1}}{u_{0}} .
$$

Notice that this last formula only involves quantities defined on the domain $\Omega_{+}$and boundary quantities computed at the previous step on the domain $\Omega_{-}$. Consequently it can be used for defining the scheme on the artificial boundary.

Once the approximate solution $\left(U_{h, I, k}^{+, n+1}, \zeta_{i, k}^{+, n+1}\right)$ in $\Omega_{+}$has been computed, we have to define the quantities

$$
B_{j, k}^{U_{h},-, n+1} \simeq f_{\left(z_{j}-\frac{\Delta z}{2}, z_{j}+\frac{\Delta z}{2}\right)} \mathcal{B}_{-}^{U_{h}} U_{h,+}^{n+1} .
$$

Indeed these quantities will be necessary for computing the approximate solution at step $n+2$ in the domain $\Omega_{-}$. For this we simply use the identity (3.28) and set

$$
B_{j, k}^{U_{h},-, n+1}:=-B_{j, k}^{U_{h},+, n}+2 \frac{\alpha}{\sqrt{\varepsilon}} A U_{h, 0, j, k}^{+, n+1} .
$$

Note that only the components of the velocity are concerned by the transmission problem in $\Omega^{-}$. The derivation of the scheme in the domain $\Omega^{-}$is similar.

6.2. Numerical optimization of the transmission conditions. In this section we are interested in the optimization of the transmission conditions (3.27) with respect to the free parameters $\alpha$ and $\beta$. More precisely, we minimize with respect to $(\alpha, \beta)$ the number of iterations required by the algorithm for reaching a given precision . This optimization problem is quite complex in our framework so we present a numerical strategy to reach the optimum. Note that in the simpler case of a 1D advection diffusion equation a complete solution of the related optimization problem 
is given in [10].

We consider a test case for which all the initial conditions (velocities and perturbation of the water height) are taken equal to zero. We initialize the algorithm (3.29) with random boundary conditions on the interface and we study the convergence of the solution towards zero. This test is quite classical to study the convergence of a domain decomposition algorithm. It is interesting since the initial quantities do contain all frequencies. In all the computations the physical parameters $\mathrm{Re}$ and $\mathrm{Fr}$ are taken equal to one, we also set $L=1.5, H=1$ and $T=1.5$ for the final time. The Rossby number $\varepsilon$ remains free. For a given value of $\varepsilon$ we apply the transmission conditions (3.27) for several values of the parameters $\alpha$ and $\beta$ and we compare the number of iterations that is needed to reach a fixed $L^{2}$ error between the computed and the analytical solutions. It allows us to find an optimal pair $\left(\alpha_{o p t}, \beta_{o p t}\right)$ that minimizes this error. This first study exhibits that the influence of the parameter $\beta$ is very small. In the following this parameter will be kept equal to its theoretical value (3.26). In a second step we study the dependency of the optimal parameter $\alpha_{\text {opt }}$ with respect to the Rossby number $\varepsilon$. As opposed to the theoretical parameter $\alpha_{\text {Tay }}$ we found that the optimized parameter $\alpha_{o p t}$ does depend on $\varepsilon$ in a nontrivial way as it is illustrated in Table 1.

\begin{tabular}{|c|c|c|c|c|c|c|}
\hline Rossby number & 0.0002 & 0.0005 & 0.001 & 0.002 & 0.005 & 0.01 \\
\hline Quotient $\alpha_{\text {opt }} / \alpha_{\text {Tay }}$ & 2.78 & 1.75 & 1.32 & 1.23 & 1.52 & 1.63 \\
\hline
\end{tabular}

Table 1: Quotient $\alpha_{\text {opt }} / \alpha_{T a y}$ as a function of the Rossby number $\varepsilon$

We now present the evolution of the error on the computed solution as a function of the number of iterations of the Schwarz waveform relaxation algorithm (3.29) in both cases $\alpha=\alpha_{o p t}$ and $\alpha=\alpha_{\text {Tay }}$. In Fig. 6.1 we present the results for two different values of the Rossby number $\varepsilon: \varepsilon=10^{-3}$ and $\varepsilon=10^{-2}$. The curves $(\log$ of the error) all look like straight lines, at least after a sufficiently large number of iterations. The method appears to be more efficient when the Rossby number is smaller since the error decreases much faster in the case $\varepsilon=10^{-3}$ - Fig. 6.1 on the left. This result is consistent with our theoretical study. Indeed, the transmission conditions are derived by an asymptotic analysis in the limit of small $\varepsilon$. We also observe that for a given value of $\varepsilon$ the curves are similar for both optimized and Taylor approximation parameters even if the error decreases faster for the optimal value $\alpha_{\text {opt }}$. Moreover let us observe that to reach an error of $10^{-4}$ (that is enough for the applicability of the Schwarz waveform relaxation algorithm) both algorithms (with optimized or Taylor approximation parameter) need a very close (and quite small) number of iterations.

We compute the same test with Rossby number $\varepsilon=10^{-2}$ but with a sinusoidal initial guess (instead of the random one) for the transmission conditions. We consider two different sinusoids with one or ten periods in the space-time considered interval and we use Taylor approximation parameters $\alpha_{\text {Tay }}$ and $\beta_{\text {Tay }}$. In Fig. 6.2 the results appear to be much better for the low frequency sinusoid than for the high frequency one. The results for the high frequency sinusoid is similar to the results obtained with a random initial guess. It follows that the method is particularly well adapted to low frequency signals : the relative error is smaller than $10^{-4}$ after only two iterations.

6.3. Applicability of the method. We end this section by some remarks concerning the applicability of the method. First we have seen above that our method was better for smooth solutions. For the linearized primitive equations without forcing, 


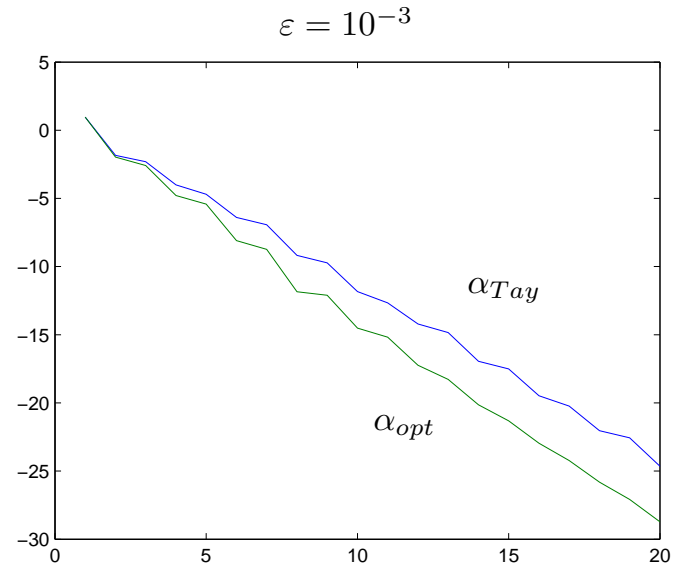

Number of iterations

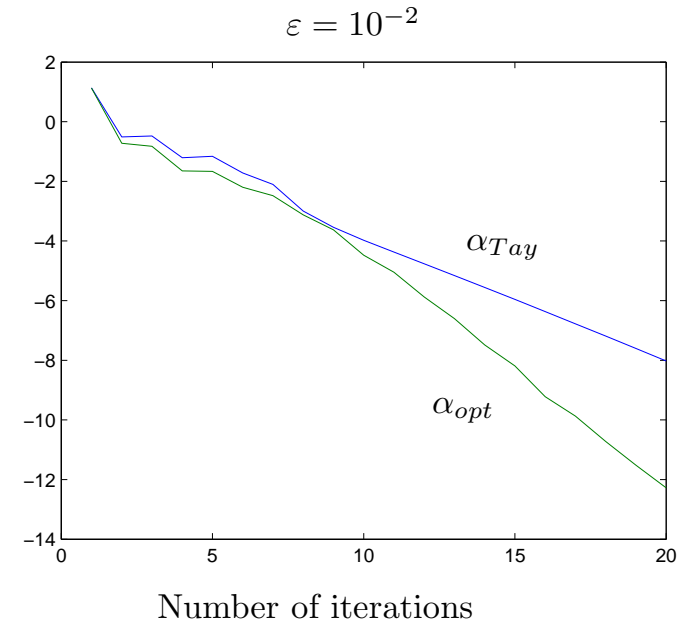

FiG. 6.1. Log of the error on the computed solution as a function of the number of Schwarz iterations for Rossby number $\varepsilon=10^{-3}$ and $\varepsilon=10^{-2}$ and for a random initial guess using the Taylor approximation parameter $\alpha_{\text {Tay }}$ (blue lines) and the optimized one $\alpha_{\text {opt }}$ (green lines)

$$
\varepsilon=10^{-2}
$$

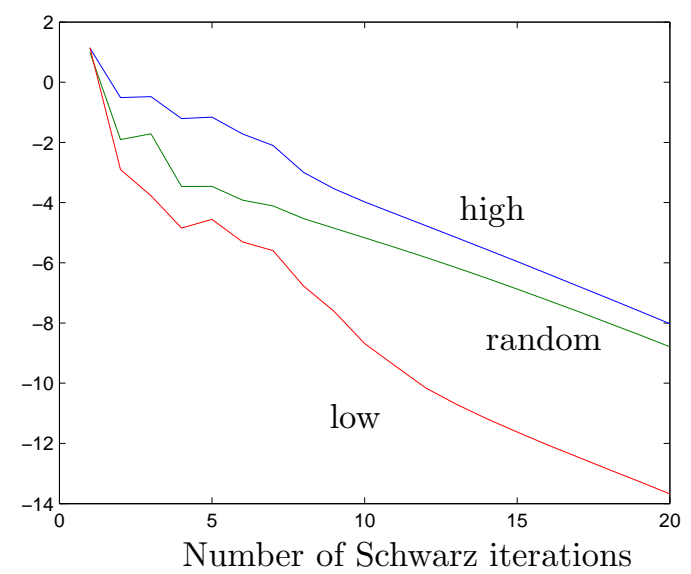

FIG. 6.2. Log of the error on the computed solution as a function of the number of Schwarz iterations for Rossby number $\varepsilon=10^{-2}$ and with optimal parameter for a low frequency signal (red line), for a high frequency signal (blue line) and for a random signal (green line)

the solutions are smooth but in realistic models, the equations contains the non-linear inertial term $U_{h} \cdot \nabla_{h} U_{h}$ and they are forced by a wind stress. In particular the small forcing scales generate turbulence. However for the numerical simulations, a subgrid scale parameterization is added to model small scales phenomena. This leads to the addition of a viscous term in the momentum equations and we can consider that the solutions are smooth.

The needed number of iterations also depends on the length of the time domain. In practice, the interval $(0, T)$ is split in $P$ time windows of lengths $T / P$. The SWR algorithm is run on each time window successively (using the solution at final time in the time window $p$ as initial data for time window $p+1)$.

REMARK 6.1. In general, if we have no useful information on the solution, we 
use a zero initial guess in the first time window. Then, for the time window $p+1$ it is more efficient to use the values of the last computed transmission conditions in time window $p$.

Finally in the numerical experiments presented above, we obtain good results for $\alpha$ close to $\frac{3}{2} \alpha_{T a y}$ and $\beta=\beta_{\text {Tay }}$. These values give good results for other experiments (not shown) with different physical parameters (but still with a small Rossby number). We are not able to provide a more accurate simple recipe for finding efficient parameters, mainly because this problem depend on a large number of variables: the physical constants, the shape and size of the domain, the smoothness of the solution, the lengths of the time windows, the step-sizes of the meshes and the type of discretization. However, a work in progress concerns the numerical optimization of the free parameters with a reasonable computational cost [B. Merlet, A simple algorithm for optimizing Schwarz methods, in preparation].

7. Conclusion. We presented in this article a new domain decomposition method for the viscous primitive equations. It involves a Schwarz waveform relaxation type algorithm with approximate transmission conditions for which we proved well-posedness. We presented a numerical optimization of the transmission conditions and we study the speed of convergence of the algorithm for several test cases. In forthcoming works we plan to increase the efficiency of the algorithm by deriving (more complex) transmission conditions based on the quasi-geostrophic asymptotic regime.

Acknowledgements. The authors thank L. Halpern and V. Martin for fruitful discussions and helpful comments. This work was partially supported by ANR program COMMA (http://www-lmc.imag.fr/COMMA/).

\section{REFERENCES}

[1] Arakawa A. \& Lamb V. Computational design of the basic dynamical processes of the UCLA general circulation model, Methods in Computational Physics, Vol. 17 (1977), pp 174-267.

[2] Blayo E. \& Debreu L. Revisiting open boundary conditions from the point of view of characteristic variables, Ocean Modelling, Vol. 9 (2005), No. 3, pp 231-252.

[3] Cailleau S., Fedorenko V., Barnier B., Blayo E. \& Debreu L. Comparison of different numerical methods used to handle the open boundary of a regional ocean circulation model of the Bay of Biscay, Ocean Modelling, Vol. 25 (2008), No. 1-2, pp 1-16.

[4] Cao C. \& Titi E.S., Global well-posedness of the three-dimensional viscous primitive equations of large scale ocean and atmosphere dynamics, Annals of Mathematics, Vol. 166 (2007), No. 1, pp 245-267.

[5] Cushman-Roisin, B. Introduction to Geophysical Fluid Dynamics, Prentice Hall (1994), pp 320.

[6] Daoud D.S. \& Gander M.J., Overlapping Schwarz waveform relaxation for convection reaction diffusion problems, Proceedings of the 13th International Conference on Domain Decomposition Methods, 2001, pp 253-260. [www.ddm.org/conferences.html]

[7] Engquist B. \& Majda A., Absorbing boundary conditions for the numerical simulation of waves, Math. Comp., Vol. 31 (1977), No. 139, pp 629-651.

[8] Gander M.J., A waveform relaxation algorithm with overlapping splitting for reaction diffusion equations, Numerical Linear Algebra with Applications, Vol. 6 (1998), pp 125-145.

[9] Gander M.J., Optimized Schwarz methods, SIAM Journal of Numerical Analysis, Vol. (2006), No., pp 699-731.

[10] Gander M.J. \& Halpern L., Optimized Schwarz Waveform Relaxation for Advection Reaction Diffusion Problems, SIAM Journal on Numerical Analysis, Vol. 45 (2007), No. 2, pp 666697.

[11] Gander M.J., Halpern L. \& Nataf F., Optimal Schwarz waveform relaxation for the one dimensional wave equation, SIAM Journal of Numerical Analysis, Vol. 41 (2003), No. 5, pp 1643-1681.

[12] Giladi E. \& Keller H.B., Space time domain decomposition for parabolic problems, Numerische Mathematik, Vol. 93 (2002), No. 2, pp 279-313. 
[13] Halpern L., Artificial boundary conditions for the advection diffusion equations, Math. Comp., Vol. 174 (1986), pp 425-438.

[14] Halpern L., Artificial boundary conditions for incompletely parabolic perturbations of hyperbolic systems, SIAM Journal on Math. Anal., Vol. 22 (1991), No. 5, pp 1256-1283.

[15] Halpern L. \& Japhet C., Discontinuous Galerkin and nonconforming in time optimized Schwarz waveform relaxation for heterogeneous problems, Domain Decomposition Methods in Science and Engineering, Vol. 60, (2008), pp. 211-219.

[16] Herzfeld, M., The role of numerical implementation on open boundary behaviour in limited area ocean models Ocean Modelling, Vol. 27 (2009), No. 1-2, pp 18-32.

[17] Jeltsch R. \& Pohl B., Waveform relaxation with overlapping splittings, SIAM J. Sci. Comp., Vol. 16 (1995), No. 1, pp 40-49.

[18] Jensen, T. G., Open boundary conditions in stratified ocean models, Journal of Marine Systems, Vol 16. (1998), No 3-4, pp 1-20.

[19] Lions P.L., On the Schwarz alternating method I, Chan T.F., Glowinski R., Periaux J ES Widlund O. editors, Proceedings of the 1st International Conference on Domain Decomposition Methods, SIAM, (1988).

[20] Marchesiello, P., McWilliams J. C. \& Shchepetkin, A., Open boundary conditions for long-term integration of regional oceanic models, Ocean Modelling, Vol. 3 (2001), No. 1-2, pp 1-20.

[21] Martin V., An optimized Schwarz waveform relaxation method for unsteady convection diffusion equation, Applied Numerical Mathematics, Vol. 52 (2005), No. 4, pp 401-428.

[22] Martin V., A Schwarz Waveform Relaxation Method for the Viscous Shallow Water Equations, Domain Decomposition Methods in Science and Engineering, Vol. 40 (2005), pp 653-660.

[23] Martin, V., Schwarz waveform relaxation algorithms for the linear viscous equatorial shallow water equations, SIAM Journal on Scientific Computing, Vol. 31 (2009), No. 5, pp 35953625 .

[24] Quarteroni A. \& Valli A., Domain Decomposition Methods for PDEs, Oxford Science Publications, London, (1999).

[25] Schwarz H.A., Über einen Grenzübergang durch alternierendes Verfahren, Vierteljahrschrift der Naturforschenden Gesellschaft in Zürich, Vol. 15 (1870), pp 272-286.

[26] Temam R. \& Tribbia J., Computational methods for the oceans and the atmosphere, Ciarlet P.G. General Editor, Special volume of the Handbook of numerical analysis, Elsevier, Amsterdam, (2008).

[27] Temam R. \& Ziane M., Some mathematical problems in geophysical fluid dynamics, Friedlander S. \& Serre D. editors, Handbook of Mathematical Fluid Dynamics, Vol. 3, Elsevier, (2004).

[28] Toselli A. \& Widlund O., Domain decomposition methods - Algorithms and theory, Series in Computational Mathematics, Vol. 34, Springer, (2004).

[29] Zisowsky, A. \& Ehrhardt, M., Discrete transparent boundary conditions for parabolic systems, Mathematical and Computer Modelling, Vol. 43 (2006), No 3-4, pp 294-309. 